2019-4

\title{
Deciphering the transport of elastic filaments by antagonistic motor proteins
}

\author{
Stephanie Portet \\ University of Manitoba, Stephanie.Portet@umanitoba.ca \\ Cecil Leduc \\ Institut Pasteur, cleduc@pasteur.fr \\ Sandrine Etienne-Manneville \\ Institut Pasteur, setienne@pasteur.fr \\ J. C. Dallon \\ dallon@math.byu.edu
}

Follow this and additional works at: https://scholarsarchive.byu.edu/facpub

Part of the Mathematics Commons, and the Other Physical Sciences and Mathematics Commons

\section{Original Publication Citation}

Physical Review E

\section{BYU ScholarsArchive Citation}

Portet, Stephanie; Leduc, Cecil; Etienne-Manneville, Sandrine; and Dallon, J. C., "Deciphering the transport of elastic filaments by antagonistic motor proteins" (2019). Faculty Publications. 3687.

https://scholarsarchive.byu.edu/facpub/3687

This Peer-Reviewed Article is brought to you for free and open access by BYU ScholarsArchive. It has been accepted for inclusion in Faculty Publications by an authorized administrator of BYU ScholarsArchive. For more information, please contact ellen_amatangelo@byu.edu. 


\title{
Deciphering the transport of elastic filaments by antagonistic motor proteins
}

\author{
Stéphanie Portet \\ Department of Mathematics, University of Manitoba, Winnipeg, MB, Canada* \\ Cécile Leduc and Sandrine Etienne-Manneville \\ Polarity, Cell migration and Cancer Lab, Institut Pasteur, Paris, France \\ John Dallon \\ Department of Mathematics, Brigham Young University, Provo, Utah, USA
}

(Dated: March 31, 2019)

\begin{abstract}
Intermediate filaments are long elastic fibres that are transported by microtubule-associated motor proteins kinesin and dynein inside the cell. How elastic filaments are efficiently transported by antagonistic motors is not well understood and difficult to measure with current experimental techniques. Adapting the tug-of-war paradigm for vesicle-like cargos, we develop a mathematical model to describe the motion of an elastic filament punctually bound to antagonistic motors. As observed in cells, up to 3 modes of transport are obtained; dynein-driven retrograde, kinesin-driven anterograde fast motions and a slow motion. Motor properties and initial conditions that depend on intracellular context, regulate the transport of filaments. Filaments elasticity is found to affect both the mode and the efficiency of transport. We further show that the coordination of motors along the filament emerges from the interplay between intracellular context and elastic properties of filaments.
\end{abstract}

PACS numbers: 87.16.Nn,87.16.Ln

Keywords: Molecular motors, Intracellular transport, Intermediate filaments

\footnotetext{
* Stephanie.Portet@umanitoba.ca
} 


\section{INTRODUCTION}

Intermediate filament proteins assemble to form filaments that organize in networks spanning the cytoplasm. Intermediate filaments vary in lengths $[1,2]$ and can form bundles that vary in diameter [3]. They have peculiar mechanical properties as they form flexible, elastic and extensible biopolymers $[4,5]$. The persistence length of intermediate filaments, in the order of $1 \mu \mathrm{m}$, is the smallest of that of all other cytoskeletal filaments [6, 7]. Vimentin filaments were recently observed to be elastic with an elastic constant which depends on the loading rate [4]. Moreover, the force-strain curves display three behaviours: an initial linear response followed by a plateau and then an increase in stiffness [4]. Furthermore, elastic properties vary with bundle diameter [8]. Filaments can be stretched to up to 3 times their rest length without breaking in vitro [5].

Intermediate filaments are involved in a wide range of cellular functions including division, apoptosis, adhesion and migration [9]. Their functions rely on their dynamic properties and spatio-temporal distribution in cells. The dynamic turnover of the intermediate filament network in cells depends on assembly/disassembly, annealing/fission and motor-driven transport [10]. Different modes of transports driven by processive motor proteins, such as dynein and kinesin-1 have been reported $[11,12]$. Rearrangements of the intermediate filament network are controlled by post-translational modifications that modulate their assembly and association with partner proteins [13] and by the regulation of microtubule driven transports $[14,15]$ (See Supplemental Material at [URL] for movies).

The core of this work is to understand how an elastic filament that is punctually attached along its length to antagonistic motors can be efficiently transported along microtubule tracks in both directions. How do motors coordinate along the length of filaments? Do filament elastic properties affect the efficiency or the direction of transport? These questions are difficult to address with current experimental procedures; hence, a mathematical model is developed to decipher plausible mechanisms that can be further investigated experimentally. A caricature of the mode of microtubule-dependent transport considered here is given in Figure 1 (See Supplemental Material at [URL] for movies of experimental observations). This type of transport is observed for type III filaments in astrocytes and fibroblasts (see,

e.g. $[12,14]$ and Supplemental Material at [URL] for movies), for neurofilaments in neurons [16], and for keratins in epithelial cells [17]. 
Mathematical models of bidirectional transport of cargo by opposite motor proteins have been previously developed (see, for instance, [18-21]). In these cases, cargoes generally consist in vesicle-like structures that can vary in size and properties. Different mechanisms for interactions between motors have been proposed such as a tug-of-war or some modes of motor activation [20]. These models identify the conditions for transport directions, end of run, pausing and rescue from pausing. Here, a model of coupled cargoes, called nodes, is proposed to uncover mechanisms of motor coordination along a filament and the emergence of filament directed (or polarized) transport (Figure 1). The coordination of motors is obtained when all the motors attached to microtubules and the same intermediate filament step at the same velocity. The model assumes a local tug-of-war at each node resulting from mechanical competition between antagonistic motors [18]. Moreover, as the density of motors per unit length of intermediate filaments assumed in the model is low, a mean

field approach is used; motors of same type share the load equally. Description of an elastic elongated object as nodes coupled by springs with a spring constant $\alpha$ is used to study how the local dynamics and the elastic properties affect the global behaviour of the system. The model considered here is the deterministic extension of a stochastic model developed in [22]; the deterministic extension is based on the expression of the dynamics of node velocities that further allows the full characterization of modes of motion of filaments. Hence, this deterministic approach allows a mathematical analysis and then a systematic study of the long run dynamics of filaments. Using this model, we find that the motion of independent (uncoupled) nodes depends on the initial conditions and motor properties as expected from the classic tug-of-war model [18]. However, our model also reveals that the filament elasticity (elastic coupling) induces the collective behaviour of motors along the filaments, and in some cases alters the expected behaviour of the nodes.

\section{MODEL}

An intermediate filament is modelled by a series of $N$ nodes connected by $N-1$ springs with spring constant $\alpha$ (Figure 1). The rest length of the springs between the nodes is denoted $\ell$. Each node represents a portion of filament. Up to $N_{D}$ dyneins and $N_{K}$ kinesins can bind to the nodes; $N_{D}$ and $N_{K}$ are the maximum of binding sites for dyneins and kinesins for one node. When node $i$ is bound to dyneins or kinesins its velocity is determined by the 
FIG. 1. An intermediate filament is represented by $N$ nodes connected by springs of rest length $\ell$ and spring constant $\alpha$. On each node, there are $N_{D}$ (resp. $N_{K}$ ) binding sites available for dyneins (resp. kinesins); the binding sites are pictured as coloured squares (here to illustrate $N_{D}=N_{K}=2$ ). Magenta represents kinesins and cyan represents dyneins. See online version for colour.

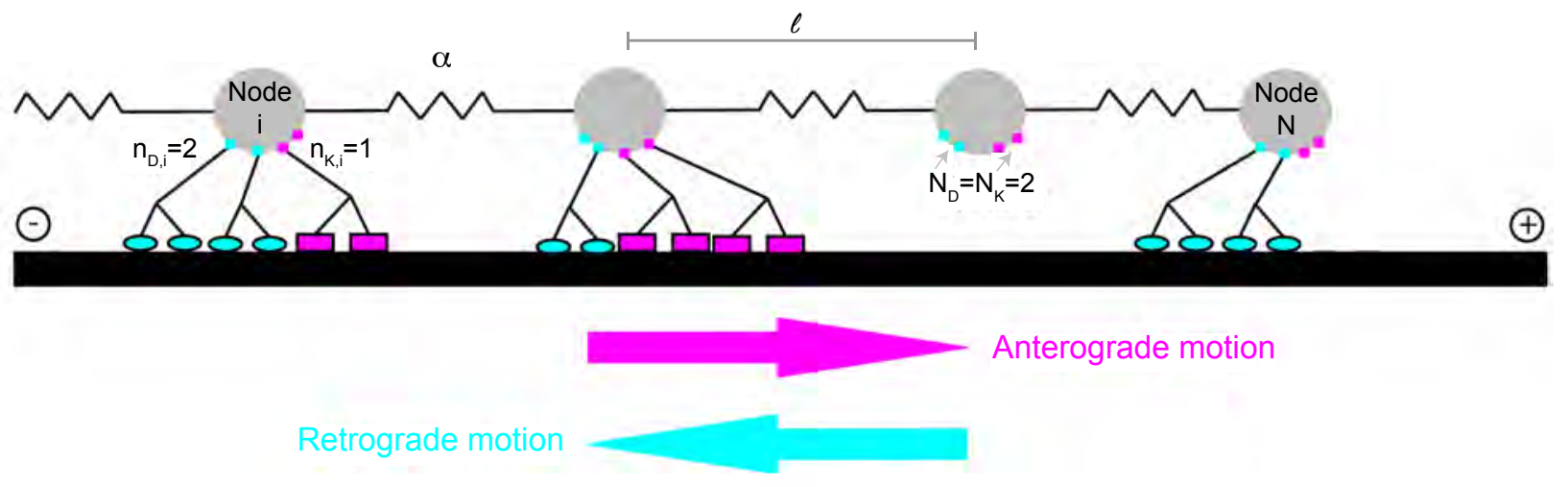

velocity of motor molecules which is a function of the load. The force-velocity relationship for dyneins and kinesins are piecewise linear functions based on previous work [23, 24] and provided in Appendix A. The model of transport of a single cargo driven by antagonistic motors along microtubules presented in $[18,19]$ is adapted in the present work for $N$ coupled cargoes. The mathematical model is based on Newton's second law of motion. The model assumes nodes exert forces on each other according to Hooke's law. The restoring force $F_{\text {res }, i}$ acting on node $i$ is proportional through $\alpha$ to the elongation from the rest distance $\ell$ between adjacent nodes, see Eq. (B14) in Appendix B. The viscous force is assumed to be proportional to the velocity of the node $i, v_{i}$. The drag coefficient $\mu$ for a node is approximated assuming a thin cylinder of length $\ell$ and filament diameter $d(\ell>d)$ moving in a fluid flowing parallel to the axis of the cylinder [25]. The inertial forces are assumed to be negligible. Hence, the equation of motion for node $i$ is given by

$$
\underbrace{\mu v_{i}}_{\text {viscous forces }}=\underbrace{n_{D, i} F_{D, i}}_{\text {forces due to dyneins }}+\underbrace{n_{K, i} F_{K, i}}_{\text {forces due to kinesins }}+\underbrace{F_{r e s, i}}_{\text {restoring forces }}
$$

where $n_{D, i}$ (resp. $n_{K, i}$ ) is the number of dyneins (resp. kinesins) attached to node $i$ and $F_{D, i}\left(\right.$ resp. $\left.F_{K, i}\right)$ is the force acting on a single dynein (resp. kinesin) attached to node $i$. Following Müller et al. [18], a tug-of-war between the motors of different types determines the motion of the cargo, and opposing motors act as loads and identical motors share equally the load. In our model, the local tug-of-war, accounting for restoring forces due to elastic coupling between nodes, occurs at each node. Note that positive forces and velocities are 
in the positive $x$-direction corresponding to the minus end-to-plus end orientation of the microtubule.

For Eq. (1) the dynamics of kinesins and dyneins attached to node $i$ is required. As it is assumed that there exists a maximal number of binding sites for dynein $\left(N_{D}\right)$ and kinesin $\left(N_{K}\right)$ at each node, the proportions of occupied binding sites are considered

$$
C_{K, i}=\frac{n_{K, i}}{N_{K}}, \quad C_{D, i}=\frac{n_{D, i}}{N_{D}}
$$

$C_{K, i}$ and $C_{D, i} \in[0,1]$, and $N_{D}=N_{K}$. The dynamics of proportions of binding sites occupied by motors of each type is described for each node $i \in\{1, \cdots, N\}$ as follows

$$
\begin{aligned}
& \frac{d C_{K, i}}{d t}=\left(1-C_{K, i}\right) \pi_{K}-\epsilon_{K} f_{K}\left(F_{K, i}\right) C_{K, i} \\
& \frac{d C_{D, i}}{d t}=\left(1-C_{D, i}\right) \pi_{D}-\epsilon_{D} f_{D}\left(F_{D, i}\right) C_{D, i}
\end{aligned}
$$

where $\epsilon_{\bullet} f_{\bullet}(\cdot)$ are the force-dependent off rates with the load-free dissociation rates $\epsilon_{\bullet}$, and

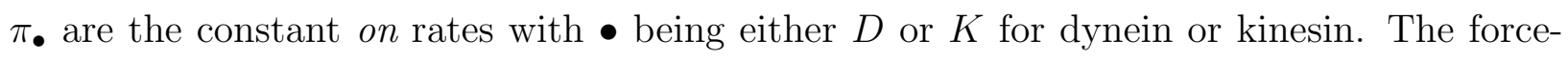
dependent parts of detachment rates $f_{K}\left(F_{K, i}\right)$ and $f_{D}\left(F_{D, i}\right)$ for kinesins and dyneins are functions of $F_{K, i}$ and $F_{D, i}$, which can be expressed as functions of $v_{i}$ as shown in equations (B8) and (B9) in Appendix B. Hence, the functions $f_{\bullet}\left(F_{\bullet}, i\right)$ can be expressed as $f_{\bullet}\left(v_{i}\right)$ and take different forms depending on the motor properties [26, 27]; here, two types of profiles are considered, the exponential profiles for kinesins (B10) or dyneins (B11) and catch-bound profile for dyneins (B12) [26, 28-30]. All profiles satisfy $f_{\bullet}(v)>0$ for $v \in\left[-v_{f D}, v_{f K}\right]$ where $-v_{f D}$ is the no-load velocity of dynein and $v_{f K}$ is the no-load velocity of kinesin.

Thus, for each node $i \in\{1, \cdots, N\}$, two equations describe the evolution of proportions of kinesin and dynein binding sites occupied and one equation following (B18) gives the evolution of the node velocity

$$
\begin{aligned}
\frac{d C_{K, i}}{d t} & =g_{K}\left(C_{K, i}, C_{D, i}, v_{i}\right) \\
\frac{d C_{D, i}}{d t} & =g_{D}\left(C_{K, i}, C_{D, i}, v_{i}\right) \\
\frac{d v_{i}}{d t} & =g\left(C_{K, i}, C_{D, i}, v_{i-1}, v_{i}, v_{i+1}\right)
\end{aligned}
$$

where $g_{K}\left(C_{K, i}, C_{D, i}, v_{i}\right), g_{D}\left(C_{K, i}, C_{D, i}, v_{i}\right)$ and $g\left(C_{K, i}, C_{D, i}, v_{i-1}, v_{i}, v_{i+1}\right)$ are piecewise functions defined over four velocity intervals resulting in four regimes (Figure 2): 


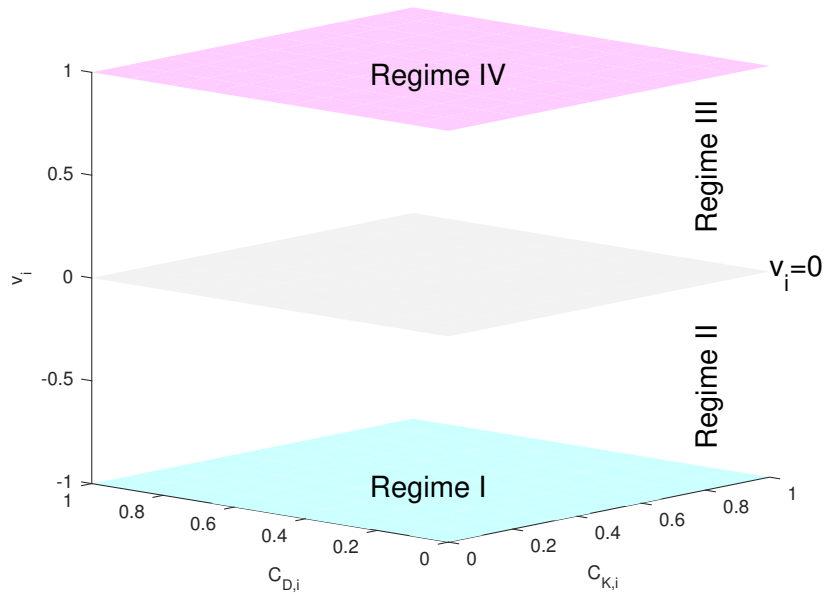

FIG. 2. Four regimes for each node $i, i \in\{1, \cdots, N\}$. In Regime I, node $i$ moves at the maximal negative velocity (no-load velocity) of dyneins, $v_{i}=-v_{f D}$. In Regime II (resp. III), node $i$ moves at an intermediate negative (resp. positive) velocity, $-v_{f D}<v_{i}<0$ (resp. $0 \leq v_{i}<v_{f K}$ ). In Regime IV, node $i$ moves at the maximal positive velocity (no-load velocity) of kinesins, $v_{i}=v_{f K}$.

- Regime I: $v_{i}=-v_{f D}$, node $i$ moves at the maximal negative velocity (no-load velocity) of dyneins,

- Regime II: $-v_{f D}<v_{i}<0$, node $i$ moves at an intermediate negative velocity,

- Regime III: $0 \leq v_{i}<v_{f K}$, node $i$ moves at an intermediate positive velocity,

- Regime IV: $v_{i}=v_{f K}$, node $i$ moves at the maximal positive velocity (no-load velocity) of kinesins.

Hence, the model for the motion of a filament composed of $N$ nodes takes the form of $3 N$-dimensional systems defined over 4 regimes. The detailed derivation of the model is given in Appendix B. The systems constituting the model are now given for the four regimes. In Regime I, the model equations are, for all nodes $i \in\{1, \cdots, N\}$

$$
\begin{aligned}
\frac{d C_{K, i}}{d t} & =\left(1-C_{K, i}\right) \pi_{K}-\epsilon_{K} f_{K}\left(-v_{f D}\right) C_{K, i}, \quad C_{K, i}\left(t_{0}\right) \in[0,1] \\
\frac{d C_{D, i}}{d t} & =\left(1-C_{D, i}\right) \pi_{D}-\epsilon_{D} C_{D, i}, \quad C_{D, i}\left(t_{0}\right) \in[0,1] \\
\frac{d v_{i}}{d t} & =0, \quad v_{i}\left(t_{0}\right)=-v_{f D}
\end{aligned}
$$


where $t_{0} \geq 0$ is the initial time. In Regime IV, the model equations are, for all nodes $i \in\{1, \cdots, N\}$

$$
\begin{aligned}
\frac{d C_{K, i}}{d t} & =\left(1-C_{K, i}\right) \pi_{K}-\epsilon_{K} C_{K, i}, \quad C_{K, i}\left(t_{0}\right) \in[0,1] \\
\frac{d C_{D, i}}{d t} & =\left(1-C_{D, i}\right) \pi_{D}-\epsilon_{D} f_{D}\left(v_{f K}\right) C_{D, i}, \quad C_{D, i}\left(t_{0}\right) \in[0,1] \\
\frac{d v_{i}}{d t} & =0, \quad v_{i}\left(t_{0}\right)=v_{f K} .
\end{aligned}
$$

Note that, in Regime I and IV, the 3N-dimensional System (2) and (3) can be reduced to $N$ 2-dimensional non-homogeneous linear systems in $C_{K, i}$ and $C_{D, i}$ as the constant velocities $v_{i}$ result in the effective decoupling of the $N$ nodes. These limiting Regime I and IV result from the model assumption that there is a maximum forward velocity for both motor molecules (Figure 14). Finally, in Regime II and III, the model equations are the following $3 N$-dimensional systems expressed with the appropriate values for $v_{0 K, i}$ and $v_{0 D, i}$ as defined in Table I:

$$
\begin{aligned}
\frac{d C_{K, 1}}{d t} & =\left(1-C_{K, 1}\right) \pi_{K}-\epsilon_{K} f_{K}\left(v_{1}\right) C_{K, 1} \\
\frac{d C_{D, 1}}{d t} & =\left(1-C_{D, 1}\right) \pi_{D}-\epsilon_{D} f_{D}\left(v_{1}\right) C_{D, 1} \\
\frac{d v_{1}}{d t} & =\frac{\alpha\left(v_{1}-v_{2}\right)+\left(1-v_{1} / v_{0 K, 1}\right) \frac{d C_{K, 1}}{d t} N_{K} F_{s K}+\left(1+v_{1} / v_{0 D, 1}\right) \frac{d C_{D, 1}}{d t} N_{D} F_{s D}}{C_{K, 1} N_{K} F_{s K} / v_{0 K, 1}-C_{D, 1} N_{D} F_{s D} / v_{0 D, 1}-\mu} \\
& \ldots \\
\frac{d C_{K, i}}{d t} & =\left(1-C_{K, i}\right) \pi_{K}-\epsilon_{K} f_{K}\left(v_{i}\right) C_{K, i} \\
\frac{d C_{D, i}}{d t} & =\left(1-C_{D, i}\right) \pi_{D}-\epsilon_{D} f_{D}\left(v_{i}\right) C_{D, i} \\
\frac{d v_{i}}{d t} & =\frac{\alpha\left(2 v_{i}-v_{i-1}-v_{i+1}\right)+\left(1-v_{i} / v_{0 K, i}\right) \frac{d C_{K, i}}{d t} N_{K} F_{s K}+\left(1+v_{i} / v_{0 D, i}\right) \frac{d C_{D, i}}{d t} N_{D} F_{s D}}{C_{K, i} N_{K} F_{s K} / v_{0 K, i}-C_{D, i} N_{D} F_{s D} / v_{0 D, i}-\mu} \\
& \cdots \\
\frac{d C_{K, N}}{d t} & =\left(1-C_{K, N}\right) \pi_{K}-\epsilon_{K} f_{K}\left(v_{N}\right) C_{K, N} \\
\frac{d C_{D, N}}{d t} & =\left(1-C_{D, N}\right) \pi_{D}-\epsilon_{D} f_{D}\left(v_{N}\right) C_{D, N} \\
\frac{d v_{N}}{d t} & =\frac{\alpha\left(v_{N}-v_{N-1}\right)+\left(1-v_{N} / v_{0 K, N}\right) \frac{d C_{K, N}}{d t} N_{K} F_{s K}+\left(1+v_{N} / v_{0 D, N}\right) \frac{d C_{D, N}}{d t} N_{D} F_{s D}}{C_{K, N} N_{K} F_{s K} / v_{0 K, N}-C_{D, N} N_{D} F_{s D} / v_{0 D, N}-\mu}
\end{aligned}
$$

where $F_{s K}$ (resp. $F_{s D}$ ) is the stall force for kinesin (resp. dynein). Parameter values are provided in Table II. System (4) in Regime II or III is considered with the initial conditions 


\begin{tabular}{|c|c|c|}
\hline Node $i$ in Regime & II & III \\
& $\left(-v_{f D}<v_{i}<0\right)$ & $\left(0 \leq v_{i}<v_{f K}\right)$ \\
\hline$v_{0 K, i}$ & $v_{b K}$ & $v_{f K}$ \\
$v_{0 D, i}$ & $v_{f D}$ & $v_{b D}$ \\
\hline
\end{tabular}

TABLE I. Values of $v_{0 K, i}$ and $v_{0 D, i}$ for Regime II and III. $v_{f} \bullet$ and $v_{b \bullet}$, where $\bullet$ is either $K$ or $D$ for kinesin or dynein, are the forward and backward speeds of motors. Parameter values are provided in Table II.

$C_{K, i}\left(t_{0}\right)=k_{i}$ and $C_{D, i}\left(t_{0}\right)=d_{i}$ with $k_{i}, d_{i} \in[0,1]$, depicting the proportions of occupied binding sites of kinesin and dynein on the filaments over a length $\ell$ at the initial time $t_{0} \geq 0$. These initial conditions describe the cellular environment in which the filament starts its journey. The initial condition for $v_{i}$ satisfies $(\mathrm{B} 4)$ considered with $F_{r e s, i}\left(t_{0}\right)=0$ assuming a distance between adjacent nodes equal to the rest length $\ell$ at $t_{0}$ and yields $v_{i}\left(t_{0}\right)=\frac{k_{i} N_{K} F_{s K}+d_{i} N_{D} F_{s D}}{k_{i} N_{K} F_{s K} / v_{0 K, i}-d_{i} N_{D} F_{s D} / v_{0 D, i}-\mu}$, for all $i \in\{1, \cdots, N\}$. Node $i$ starts in Regime II (resp. III) if $-v_{f D}<v_{i}\left(t_{0}\right)<0\left(\right.$ resp. $\left.0 \leq v_{i}\left(t_{0}\right)<v_{f K}\right)$.

Furthermore, as motor properties for detachment are still not entirely determined experimentally [27], we investigate how different off rate profiles $f_{\bullet}$ modify the dynamics. The complete model, composed of System (2), (3) and (4), is considered with both exponential off rates for dyneins and kinesins as defined in (B11) and (B10), or catch-bond off rate for dyneins (B12) and exponential profile for kinesins (B10). The latter combination of off rates is also called mixed rates.

\section{ANALYSIS}

In Regime I and IV, the $N$ decoupled planar non-homogeneous linear systems deduced from System (2) and (3) have unique solutions that are bounded; it can be shown that $[0,1] \times[0,1]$ is invariant under the flow of (2) and (3). Solutions started in Regime I (resp. IV) cannot leave this regime (Figure 2). The vector field of System (4) is $C^{1}$ except on the switching hyperplane defined by $v_{i}=0$, where the vector field is only $C^{0}$. That switching hyperplane is not invariant and solutions starting in Regime II can enter Regime III and vice versa. System (4) that describes the dynamics in Regime II or III has unique solutions except maybe on the switching hyperplane. Furthermore, it can be shown that 


\begin{tabular}{|c|c|c|c|}
\hline Parameter (unit) & Description & Kinesin & Dynein \\
\hline$v_{f \bullet}(\mu m / s)$ & Forward speed of motors & 1 & 1 \\
\hline$v_{b \bullet}(\mu m / s)$ & Backward speed of motors & 0.006 & 0.006 \\
\hline$F_{d \bullet}(\mathrm{pN})$ & Detachment force & 3 & 3 \\
\hline$F_{s \bullet}(\mathrm{pN})$ & Stall force & -6 & 7 \\
\hline \multirow{2}{*}{$\left|F_{s \bullet}\right| / F_{d \bullet}$} & \multirow[t]{2}{*}{ Strength of motors } & $-6 \mid / 3($ Strong $)$ & $|7| / 3$ (Strong) \\
\hline & & $|-3| / 3($ Weak $)$ & $|3| / 3$ (Weak) \\
\hline$\epsilon_{\bullet}\left(\mathrm{s}^{-1}\right)$ & Free-load dissociation rate & 1 & 0.25 \\
\hline$\pi_{\bullet}\left(\mathrm{s}^{-1}\right)$ & Attachment rate & 5 & 1.5 \\
\hline$\kappa_{\bullet}=\epsilon_{\bullet} / \pi_{\bullet}$ & Desorption constant & 0.2 & 0.16 \\
\hline$N \bullet$ & Maximal number of binding sites & $2-16$ & $2-16$ \\
\hline$N$ & Number of nodes & \multicolumn{2}{|l|}{$\geq 1$} \\
\hline$\alpha(N / \mu m)$ & Spring constant & \multicolumn{2}{|l|}{$3.45 \times 10^{-9} / N$} \\
\hline$\ell(\mu m)$ & Rest length of spring & \multicolumn{2}{|l|}{0.5} \\
\hline$\mu(N s / \mu m)$ & Drag coefficient for a node & \multicolumn{2}{|l|}{$1.2 \times 10^{-12}$} \\
\hline
\end{tabular}

TABLE II. Base parameter values used unless otherwise mentioned in the model, $\bullet$ is either $K$ or $D$ for kinesin or dynein. Base values for motor parameters follow [18, 19, 28, 31]. The value for $\mu$ is obtained from Eq. (2) in [25]; $\mu=\frac{3 \pi \ell \eta}{\ln (p)+v}$ where $p=\ell / d$ with $d=0.01 \mu m$ is the intermediate filament diameter and $v=0.312+0.565 p^{-1}-0.1 p^{-2}$. The cytosol viscosity is set to be $\eta=1.08$ $\mathrm{Pa} \cdot \mathrm{s}$, about 1000 times higher than water. Using Hooke's law, $F=\alpha \Delta L$, there exists a relationship between the spring constant $\alpha$ and the Young's modulus $\mathcal{Y}(=$ stress $/$ strain $=(F / A) /(\Delta L / L))$ given by $\alpha=\mathcal{Y} A / L$ with $L=\ell$. The elastic modulus of vimentin has been estimated to be 8-9 MPa [32], $900 \mathrm{MPa}$ by [33], and 6.5 MPa for another keratin-like based filament [7]. For a 10 percent change of length with forces on the order of $0.18 \mathrm{pN}$ a Young's modulus of $22 \mathrm{MPa}$ is needed. This lies in the range of experimentally measured values and compares well with values used in [4]. Thus, the base value for $\alpha$ is $3.45 \times 10^{-9} / N$.

in Regime II $\left.\frac{d v_{i}}{d t}\right|_{v_{i}=-v_{f D}}<0$ and in Regime III $\frac{d v_{i}}{d t}{ }_{v_{i}=v_{f K}}>0$; hence, solutions started in Regime II can enter in Regime I and solutions started in Regime III can enter in Regime IV.

Summing up, solutions initiated in Regime II or III can travel between these 2 regimes 
and approach some of their stable features/objects (equilibrium points or cycles) or might eventually enter in one of the limiting Regime I or IV and be trapped there. Due to the complexity and dimensionality of the model, we first characterize the dynamics in each regime; to do so, each system from (2) to (4) is studied separately.

\section{A. Regime I and IV}

In this section the long run behaviour of solutions starting or ending up in Regime I and IV (the limiting regimes) is characterized. It is shown that there exists a unique equilibrium which is the same for all nodes

$$
\left(C_{K, i}^{*}, C_{D, i}^{*}, v_{i}^{*}\right)= \begin{cases}\left(C_{K I}^{*}, C_{D I}^{*}, v_{I}^{*}\right)=\left(\frac{1}{1+\kappa_{K} f_{K}\left(-v_{f D}\right)}, \frac{1}{1+\kappa_{D}},-v_{f D}\right), & \text { in Regime I } \\ \left(C_{K I V}^{*}, C_{D I V}^{*}, v_{I V}^{*}\right)=\left(\frac{1}{1+\kappa_{K}}, \frac{1}{1+\kappa_{D} f_{D}\left(v_{f K}\right)}, v_{f K}\right), \text { in Regime IV }\end{cases}
$$

that is globally asymptotically stable (GAS). The * denote steady state values and the constants $\kappa_{\bullet}=\epsilon_{\bullet} / \pi_{\bullet}$ are desorption constants (detachment/attachment). The proof of this result is given in Appendix $\mathrm{C}$.

In other words, if all nodes of a filament start with the initial velocity $-v_{f D}$ in Regime I (resp. $v_{f K}$ in Regime IV) or enter from Regime II (resp. III) to Regime I (resp. IV), then nodes move at the same maximal velocity and stay in the limiting Regime I (resp. IV). In the long run, when both off rates are exponential, in Regime I (resp. IV), kinesins (resp. dyneins) are detached from the filament, $C_{K I}^{*} \approx 0$ (resp. $\left.C_{D I V}^{*} \approx 0\right)$, and the proportion of occupied binding sites for dyneins (resp. kinesin) eventually approaches $C_{D I}^{*}=$ $1 /\left(1+\kappa_{D}\right)\left(\operatorname{resp} . C_{K I V}^{*}=1 /\left(1+\kappa_{K}\right)\right)$. When mixed off rates are considered, in Regime IV, dyneins are still attached to filaments, and the proportion of dynein binding sites occupied, $C_{D I V}^{*} \approx 1 /\left(1+\kappa_{D} 0.4 \exp \left(F_{s D} / F_{d D}\right)\right)$, depends on the strength of dyneins $F_{s D} / F_{d D}$ where $F_{s D}$ and $F_{d D}$ are the stall and detachment forces for dynein. Note that, in Regime I and IV, the filament properties (the length $N$ and elasticity $\alpha$ ) play no role in the dynamics. In Regime I or IV, the asymptotic dynamics is completely characterized; the filament moves steadily at the maximal velocity of motors winning the competition. 


\section{B. Regime II and III}

Now, System (4) is studied in isolation to characterize its specific stable features; details of the analysis are provided in Appendix D. In Regime II and III, at equilibrium, all nodes of a filament move at the same velocity $v^{*}$ satisfying

$$
0=\left(1-v^{*} / v_{0 K, i}\right) \frac{N_{K} F_{s K}}{1+\kappa_{K} f_{K}\left(v^{*}\right)}+\left(1+v^{*} / v_{0 D, i}\right) \frac{N_{D} F_{s D}}{1+\kappa_{D} f_{D}\left(v^{*}\right)}+v^{*} \mu
$$

with $\kappa_{\bullet}=\epsilon_{\bullet} / \pi_{\bullet}$. The final velocities $v^{*}$ are numerically computed for Regime II and III by taking the appropriate $v_{0 K, i}$ and $v_{0 D, i}$ as defined in Table I. The equilibrium points for System (4) in Regimes II and III are:

$$
\forall i,\left(C_{K, i}^{*}, C_{D, i}^{*}, v_{i}^{*}\right)=\left(C_{K}^{*}, C_{D}^{*}, v^{*}\right)=\left(\frac{1}{1+\kappa_{K} f_{K}\left(v^{*}\right)}, \frac{1}{1+\kappa_{D} f_{D}\left(v^{*}\right)}, v^{*}\right)
$$

As shown in Appendix D those equilibrium points are interior equilibria. To study their local stability, the linearization of System (4) is used. The sign of the real parts of the eigenvalues of the Jacobian matrix of System (4) evaluated at equilibrium is determined using Routh-Hurwitz criterion; details of the stability analysis are given in Appendix D.

Neither the mode of motion (value of final velocity, $v^{*}$ ) nor its uniqueness or diversity (number of possible final velocity, $v^{*}$ ) is accessible analytically as (5) has to be solved numerically. Numerical bifurcation analysis is then carried out to determine the possible modes of motion for filaments in Regime II and III. Solutions $v^{*}$ of equation (5) are computed using the $\mathrm{R}$ package rootSolve and the Matlab function InterX. The nature of equilibrium points is then determined by evaluating conditions (D4). Parameter values used for this theoretical study are provided in Table II [18, 19, 24, 26, 31]. Bifurcation diagrams displaying equilibrium points and their local stability as a function of motor strength, $\left|F_{s \bullet}\right| / F_{d \bullet}$, are provided in Appendix $\mathrm{F}$ and phase diagrams summarizing the information are given in Figure 3.

When both exponential off rates are used, in Regime II and III, up to five equilibrium points can be found (Appendix F and Table V); tristability or bistability is observed depending on parameter values (yellow or light green region (white or light gray region in greyscale version) of Figure 3a). Hence, in Regime II and III, with both exponential off rates, there exist up to 3 different dynamics: anterograde motion (positive fast velocities, $v^{*}=v_{I I I}^{*}$ ), slow motion (small velocities, $v^{*}=v_{S l o w}^{*}$ ) and retrograde motion (negative fast 


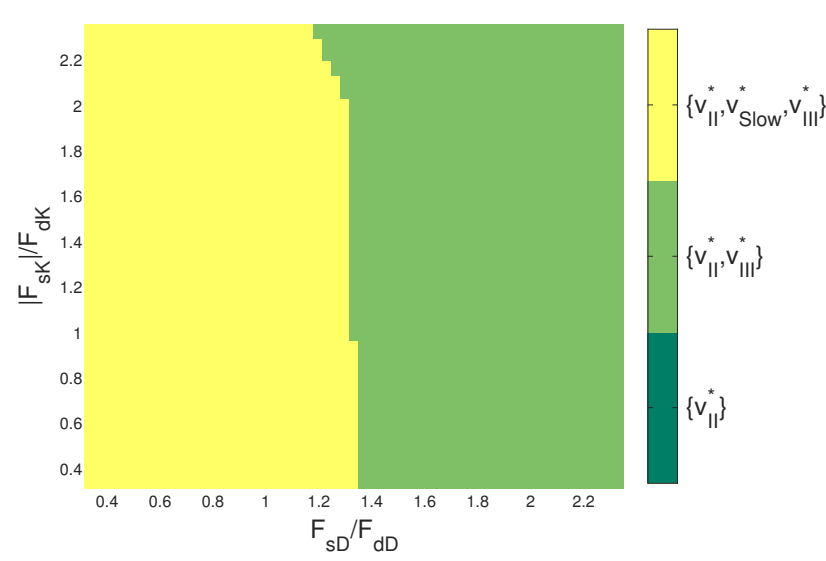

(a) Both exponential rates, $\left|F_{s} \bullet\right| / F_{d \bullet}$

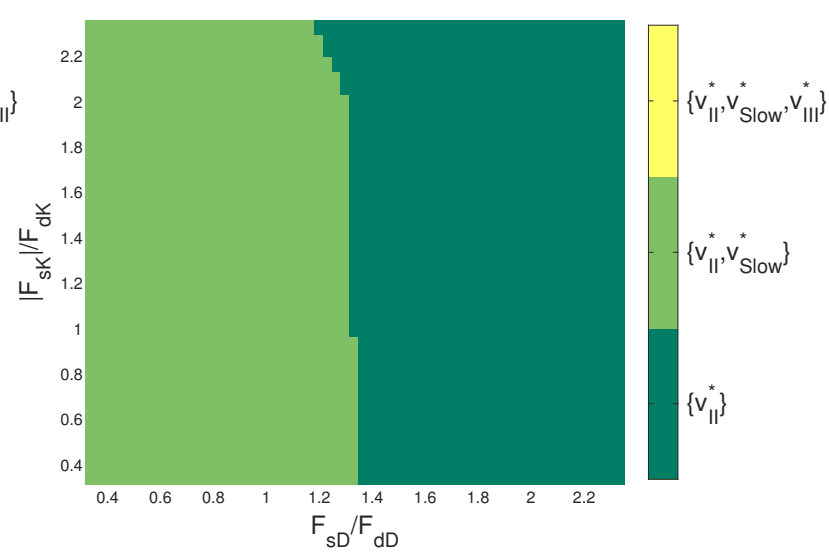

(b) Mixed rates, $\left|F_{s \bullet}\right| / F_{d \bullet}$

FIG. 3. Locally asymptotically stable equilibrium points in Regime II and III with $N_{K}=N_{D}=16$ and $N=5$ depending on strength of motors, $\left|F_{s} \bullet\right| / F_{d \bullet}$. (3a) With both exponential off rates up to 3 dynamics can be observed: anterograde motion (positive fast velocities, $v^{*}=v_{I I I}^{*}$ ), "slow" motion (small velocities, $v^{*}=v_{S l o w}^{*}$ ) and retrograde fast motion (negative velocities, $v^{*}=v_{I I}^{*}$ ). For both exponential off rates, anterograde and retrograde motions are always possible. (3b) For mixed rates, only the retrograde motion is always possible. Only slow filaments can be observed for weak dyneins, $F_{s D} / F_{d D}<1.1$.

velocities, $\left.v^{*}=v_{I I}^{*}\right)$. Retrograde and anterograde motions are always possible for parameter values considered. For anterograde (resp. retrograde) motion, the final velocity $v^{*}=v_{I I I}^{*}$ (resp. $v^{*}=v_{I I}^{*}$ ) is largely positive (resp. negative) and nodes are mainly attached to kinesins (resp. dyneins) and $C_{D}^{*} \approx 0$ (resp. $\left.C_{K}^{*} \approx 0\right)$. In slow motions, the steady state velocity $v^{*}=v_{\text {Slow }}^{*}$ is slightly positive or negative, both types of motors stay attached to the nodes, there is no winner to the competition; the tug-of-war is not resolved (Table V). For catch-bond off rate for dynein and exponential off rate for kinesin (mixed rates), in Regime II and III, only up to 3 equilibrium points are found (Table V); bistability occurs in light green region (light gray region in greyscale version) of Figure 3b. The retrograde motion, $v^{*}=v_{I I}^{*}<0$, is always possible when dynein is considered with catch-bond off rates. Slow motions can only be observed when dynein is weak $F_{s D} / F_{d D}<1.1$ and the velocities $v_{\text {Slow }}^{*}$ are slightly positive or negative (Figure 3 and Table V).

We have investigated the impact of the forward and backward speeds of motor molecules, $v_{f} \bullet$ and $v_{b \bullet}$, on the final velocity values $v_{I I}^{*}, v_{S l o w}^{*}$ and $v_{I I I}^{*}$ (Figure 4$)$. Fast velocities $\left(v_{I I}^{*}\right.$ 


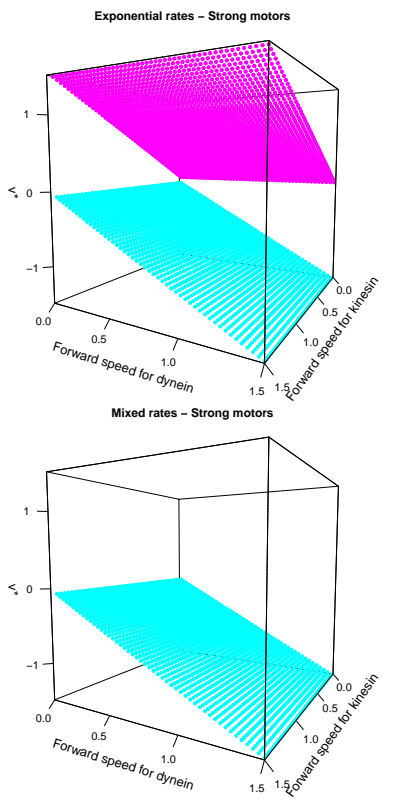

(a) Forward speeds
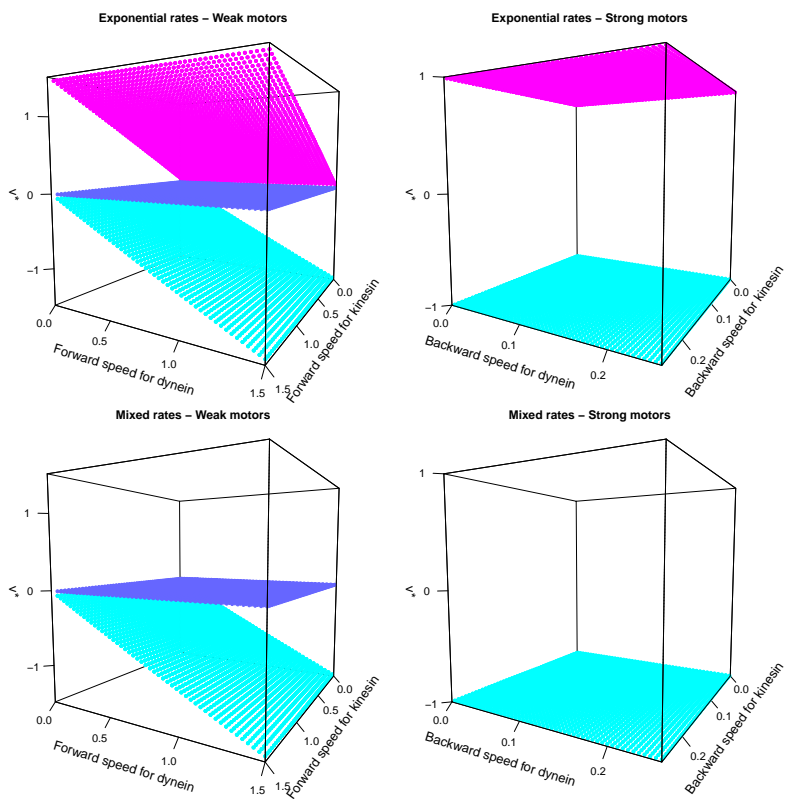

(b) Backward speeds
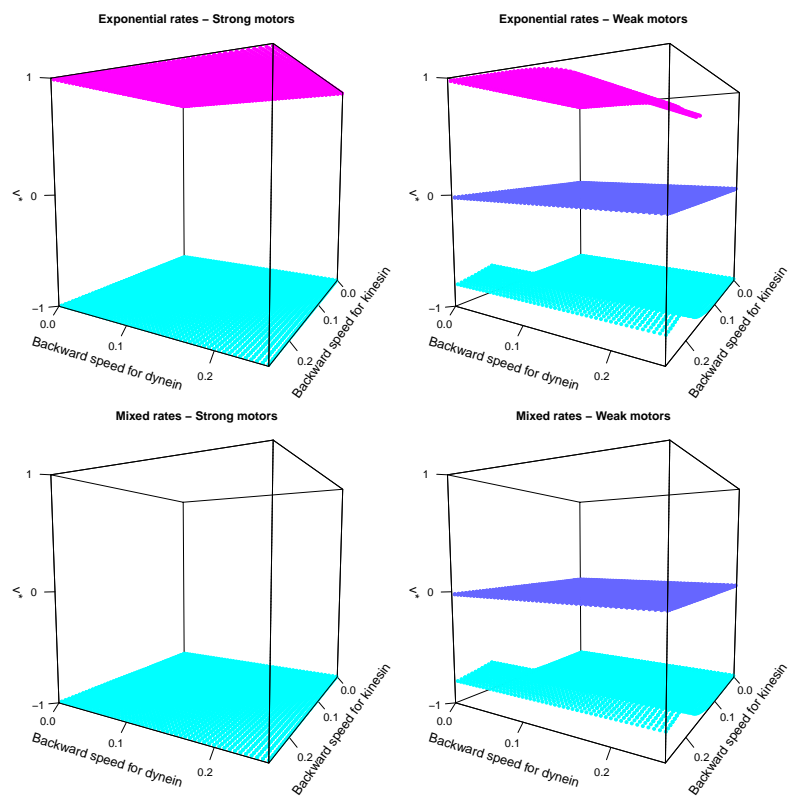

FIG. 4. Sensitivity of locally asymptotically stable equilibrium values, $v^{*}$, to values of forward and backward speeds, $v_{f}$ and $v_{b \bullet}$, in Regime II and III with $N_{K}=N_{D}=16$ and $N=2$. Units of all speeds and velocities are $\mu \mathrm{m} / \mathrm{s}$. When varying, the forward (resp. backward) speeds range in $[0.06,1.5]$ (resp. $[0.005,0.25])$. The other parameter values are constant and given in Table II. Positive fast velocities $v^{*}=v_{I I I}^{*}$ appear in magenta (dark grey), negative fast velocities $v^{*}=v_{I I}^{*}$ in cyan (light grey) and small velocities corresponding to slow motion $v^{*}=v_{S l o w}^{*}$ in purple blue (black surface near zero). Strong motors correspond to $\left|F_{s K}\right| / F_{d K}=2$ and $F_{s D} / F_{d D}=2.3$ and weak motors correspond to $\left|F_{s K}\right| / F_{d K}=F_{s D} / F_{d D}=1$.

and $\left.v_{I I I}^{*}\right)$ are found to be proportional to the maximal forward speed of the corresponding motor (4a) but they are not affected by backward speeds for strong motors $\left(\left|F_{s K}\right| / F_{d K}=2\right.$ and $\left.F_{s D} / F_{d D}=2.3\right)$. However, for weak motors $\left(\left|F_{s K}\right| / F_{d K}=1\right.$ and $\left.F_{s D} / F_{d D}=1\right)$, when $v_{f \bullet} / v_{b \bullet}<5, v_{I I}^{*}$ and $v_{I I I}^{*}$ are limited by the backward speed of the opposite motors $(4 \mathrm{~b})$. Slow motion velocities $v_{\text {Slow }}^{*}$ are not sensitive to variations in either forward or backward speeds (Figure 4).

The aim of the present work is to characterize the effect of the elastic coupling $\alpha$. Bifurcation diagrams for $N$ uncoupled nodes $(\alpha=0)$ and $N$ coupled nodes $(\alpha>0)$ are found to be similar; equilibrium points have the same values and their nature (locally asymptotically stable (LAS) or unstable) does not change (Appendix F and Table V). Recall that the 
equilibrium values defined in (6) depend on the off rate profiles, stall forces $F_{s \bullet}$, the desorption constant $\kappa_{\bullet}$, the number of binding sites $N_{\bullet}$ and the drag $\mu$. However, they do not depend on the filament properties $N$ and $\alpha$ whereas the local stability conditions (D4) rely on $N$ and $\alpha$. By considering $N$ uncoupled nodes $(\alpha=0)$, we show that the coupling cannot stabilize unstable equilibrium points and the coupling of nodes results in extra conditions to be satisfied to ensure the local stability of equilibria. The local stability conditions for the uncoupled case are included in the conditions to satisfy in the coupled case (Appendix D). Hence, the uncoupled conditions are necessary conditions for the local stability of coupled nodes. Furthermore, for the parameter values investigated, the uncoupled conditions are found to be the necessary and sufficient conditions for the local stability in coupled cases.

Summing up, in the long-run, for a filament to reach a steady velocity, all nodes of a filament move at the same velocity $v^{*}$, and all nodes have the same unique steady proportion $\left(C_{K}^{*}, C_{D}^{*}\right)$ of occupied binding sites for kinesin and dynein. Hence, we prove that a steady motion of filaments results from the coordination or collective behaviour of motors along filaments. However, due to the high dimension of the system, this analysis is not sufficient to deduce the basins of attraction of locally asymptotically stable equilibria, and completely characterize the dynamics of filaments and the effects of initial conditions and coupling. Hence, the characterization of mechanisms driving the coordination or collective behaviour of motors necessitates dynamical simulations of the complete model.

\section{RESULTS}

The complete model combining the four regimes is now considered to grasp conditions defining the filament dynamics. Numerical simulations of the complete model are carried out with the two combinations of off rates. Moreover, two values of motor strength $\left|F_{s \bullet}\right| / F_{d \bullet}$, where $F_{s}$ and $F_{d \bullet}$ are the stall and detachment forces for motors, are considered as the dynamics of systems in Regime II and III depends on the value of this ratio as shown in Figure 3. Hence, the complete model is numerically solved in four cases:

Case 1: Both exponential off rates with strong motors, $\left|F_{s K}\right| / F_{d K}=2$ and $F_{s D} / F_{d D}=2.3$;

Case 2: Both exponential off rates with weak motors, $\left|F_{s \bullet}\right| / F_{d \bullet}=1$;

Case 3: Mixed off rates with strong motors, $\left|F_{s K}\right| / F_{d K}=2$ and $F_{s D} / F_{d D}=2.3$; 
Case 4: Mixed off rates with weak motors, $\left|F_{s \bullet}\right| / F_{d \bullet}=1$.

All simulations are started with initial conditions satisfying Regime II or III (System (4)); hence, trajectories can evolve within these intermediate regimes or become trapped in one of the limiting Regime I or IV. The numerical solutions of the model are computed over a time period $\left[0, t_{\text {final }}\right]$ using the $\mathrm{R}$ package deSolve.

\section{A. The elastic properties of filaments ensure the coordination of motors}

Figures $5 \mathrm{a}-5 \mathrm{~d}$ provide the regions of initial conditions for uncoupled nodes $(\alpha=0)$ leading to specific motions. Up to 3 modes of motion are obtained: retrograde (cyan), anterograde (magenta) and slow (purple blue) motions. When the $N$ nodes are coupled $(\alpha>0)$ and start with the same initial conditions, such as $C_{K, i}(0)=k$ and $C_{D, i}(0)=d$ with $i \in\{1, \cdots, N\}$ and $k, d \in[0,1]$, the same regions of initial conditions are obtained for the coupled case (Figures $5 \mathrm{a}-5 \mathrm{~d})$. The $N$ coupled nodes are coordinated and have the same final mode of motion as an uncoupled node starting with $C_{K, 1}(0)=k$ and $C_{D, 1}(0)=d$. The coupling does not affect the direction of motion when the initial conditions are the same along the filament.

If the coordination of nodes (and so of motors) is only due to initial conditions, the behaviour of a filament (coupled nodes) can be deduced from the local behaviour of its nodes when all nodes start with initial conditions leading to similar dynamics. In other words, if all nodes of a filament started with initial conditions such as they would exhibit a retrograde, anterograde or unresolved (slow) motions when uncoupled, all nodes would have the same behaviour when coupled. These nodes are called implicitly coordinated. In Figures 5e-5h, a filament of 2 coupled nodes is considered: the colour on the horizontal (resp. vertical) axis represents the mode of motion of node 1 (resp. 2) when it starts with the initial conditions $C_{K, 1}(0)-C_{D, 1}(0)$ (resp. $\left.C_{K, 2}(0)-C_{D, 2}(0)\right)$ as found in Figures $5 \mathrm{a}-5 \mathrm{~d}$. Combining the expected motion of node 1 and 2 when they are uncoupled the light coloured regions are obtained. These regions are called regions of implicit coordination and predict the motions of the filament of 2 nodes when its nodes start with the appropriate initial conditions. When nodes of a filament start with initial conditions from white regions $\left(5 \mathrm{e}^{-}\right.$ $5 \mathrm{~h}$ ), the motion of the filament cannot be predicted from the local dynamics of its nodes. 


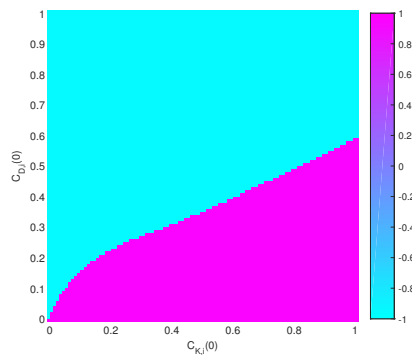

(a) Case 1

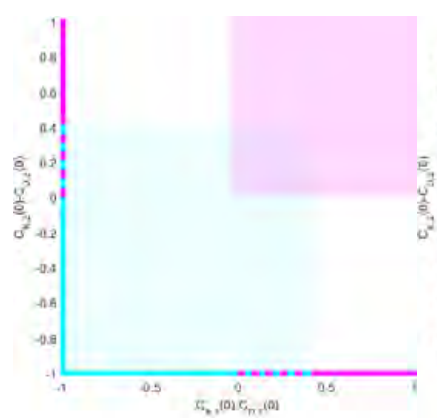

(e) Case 1

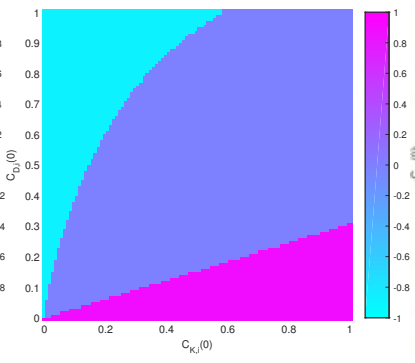

(b) Case 2

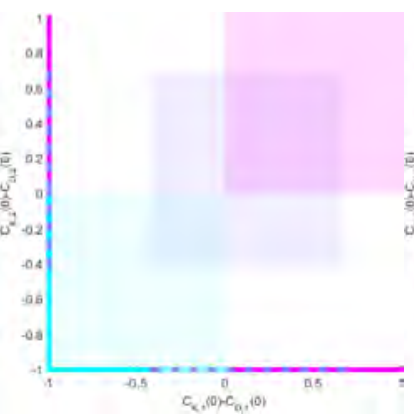

(f) Case 2

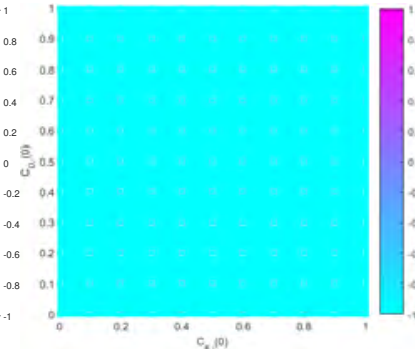

(c) Case 3

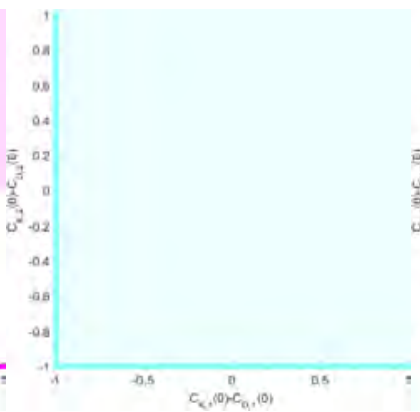

(g) Case 3

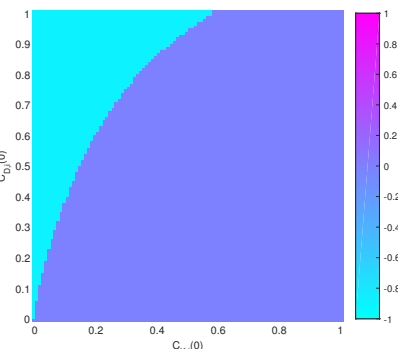

(d) Case 4

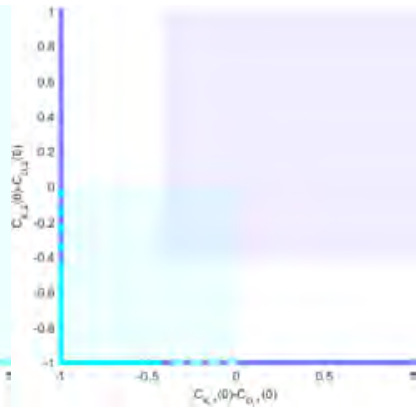

(h) Case 4

FIG. 5. Effect of initial conditions on the long run dynamics. Colour represents the final velocity $v^{*}$ of filament/node, colour bars indicate velocity values (units are $\mu \mathrm{m} / \mathrm{s}$ ). When initial conditions are in the magenta (dark grey) (resp. cyan (light grey)) region, the filament/node moves anterogradely (resp. retrogradely). Filament/node starting with initial conditions in the purple blue (middle grey) region is almost stalled (slow velocities). (5a-5d) $N$ uncoupled and $N$ coupled nodes starting with same initial conditions $C_{K, 1}(0)=\cdots=C_{K, N}(0)$ and $C_{D, 1}(0)=\cdots=C_{D, N}(0)$ have the same dynamics $\left(N_{K}=N_{D}=4\right)$. As previously mentioned, slow velocities are only possible with weak motors $(5 \mathrm{~b}$ and $5 \mathrm{~d})$. In $(5 \mathrm{e}-5 \mathrm{~h})$ light colour regions are the regions of implicit coordination for a filament of 2 nodes predicted by coupling the dynamics of 2 uncoupled nodes. See online version for colour.

To capture the effect of coupling, the dynamics of 2 uncoupled or coupled nodes is studied in detail (Figure 6). For each Case 1 to 4, 10,000 simulations with initial conditions $C_{K, i}(0)$ and $C_{D, i}(0)$ uniformly distributed in $[0,1]$ for $i \in\{1,2\}$ are carried out. For comparison purposes, for each case, simulations performed with $\alpha=0$ (6a-6d) and $\alpha$ equal to its base value (6e-6h) have the same initial conditions. Comparing Figures 5e-5h to Figure 6 shows that the regions of implicit coordination provide good predictions of the coupled motion. When nodes are not implicitly coordinated (initial conditions from white regions 


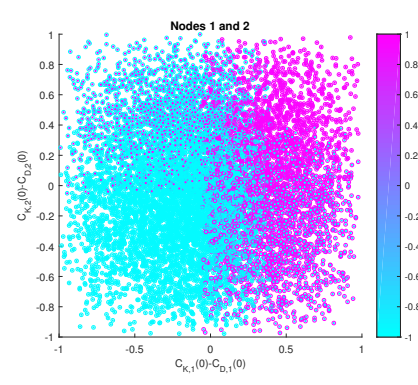

(a) Case 1 with $\alpha=0$

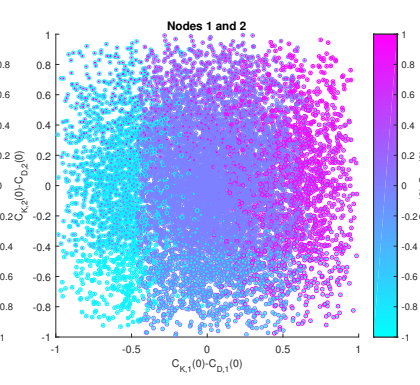

(b) Case 2 with $\alpha=0$

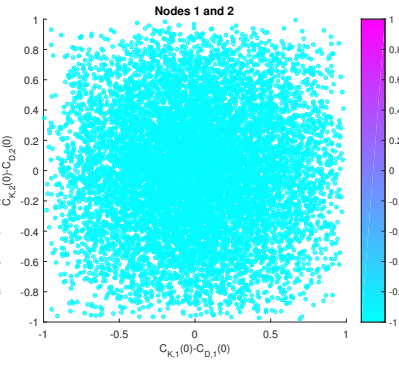

(c) Case 3 with $\alpha=0$

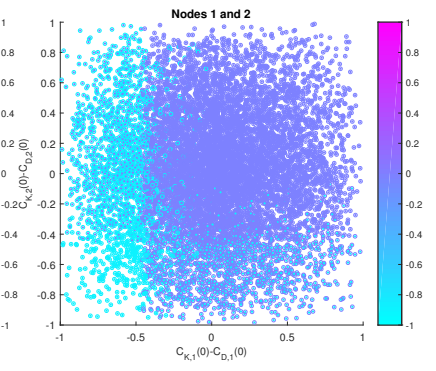

(d) Case 4 with $\alpha=0$

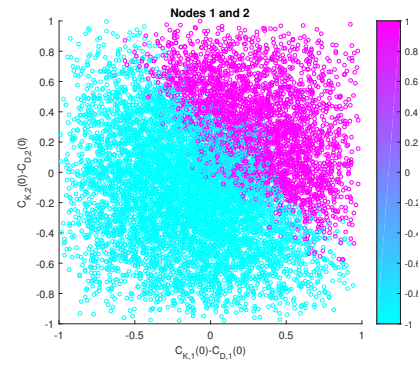

(e) Case 1 with $\alpha$

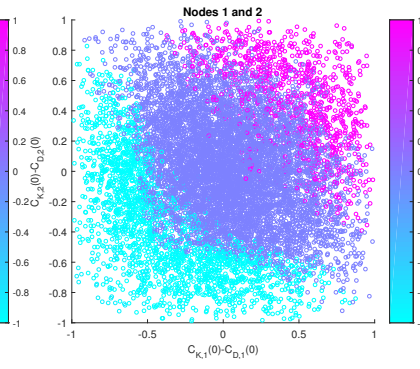

(f) Case 2 with $\alpha$

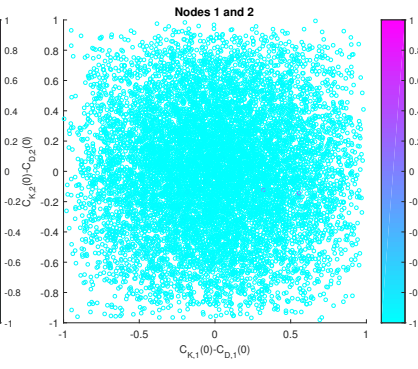

(g) Case 3 with $\alpha$

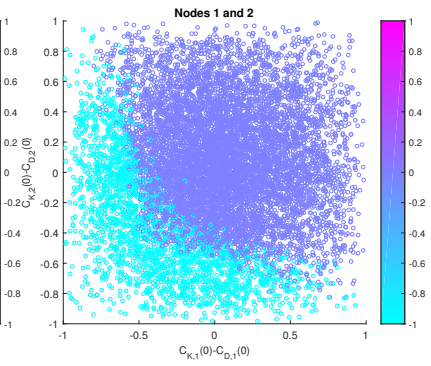

(h) Case 4 with $\alpha$

FIG. 6. Dynamics of 2 nodes with $N_{K}=N_{D}=4$ : (6a-6d) uncoupled nodes, (6e-6h) coupled nodes using the base value for $\alpha$. For each case, 10,000 simulations are performed with initial conditions $C_{K_{i}}(0)$ and $C_{D_{i}}(0)$ uniformly distributed on $[0,1]$. Each circle represents the initial conditions of the nodes of one simulation. (6a-6d) Colour of outer (resp. inner) circles represents the final velocities $v^{*}$ of node 1 (resp. node 2$)$. (6e-6h) The colour of circles is the filament final velocity $v^{*}$. Only simulations terminating with the same final velocities for both nodes are considered $\left(\left|v_{1}\left(t_{\text {final }}\right)-v_{2}\left(t_{\text {final }}\right)\right|<10^{-3}\right)$. Roughly 1 percent of the simulations do not meet the criterion and are not considered. See online version for colour.

in $5 \mathrm{e}-5 \mathrm{~h}$ or regions in $6 \mathrm{a}-6 \mathrm{~d}$ where the outer and inner circles have different colours) the elastic coupling ensures their coordination and dictates the final mode of motion (6e-6h). For illustration, trajectories of two nodes are plotted in the $C_{K, i}-C_{D, i}-v_{i}$ hyperplane (Figure 7). In Figures 7a-7d, both nodes start with initial conditions from the same region of implicit coordination, the coupling does not change the direction of motion (all anterograde). However, when coupled, nodes finish in Regime IV with the velocity $v_{I V}^{*}(7 \mathrm{~b}-7 \mathrm{~d})$ instead of finishing in Regime III with the velocity $v_{I I I}^{*}$ as they do when they are uncoupled $(\alpha=0$, 7a). The coupling does not change the value and nature of equilibria but affects their basin of attraction. In this case the coupling makes the transport more efficient. In the coupled 


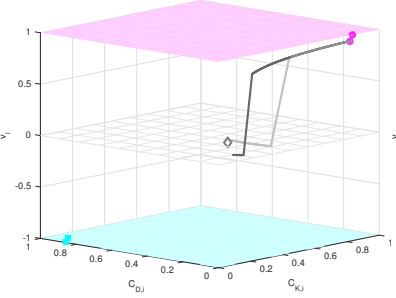

(a) Case 1, $\alpha=0$

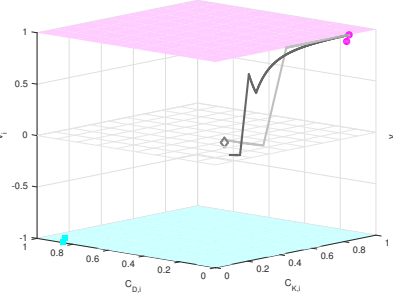

(b) Case $1,10^{-1} \alpha$

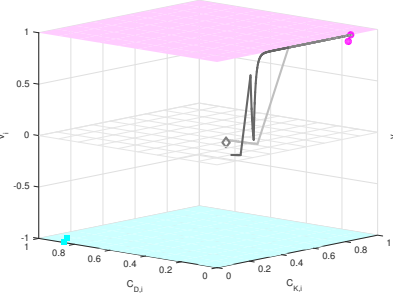

(c) Case 1, $\alpha$

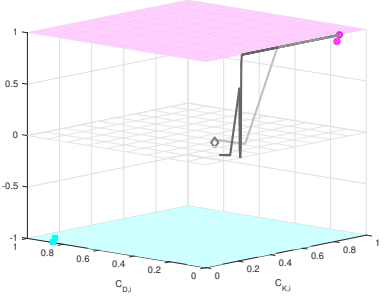

(d) Case $1,10 \alpha$

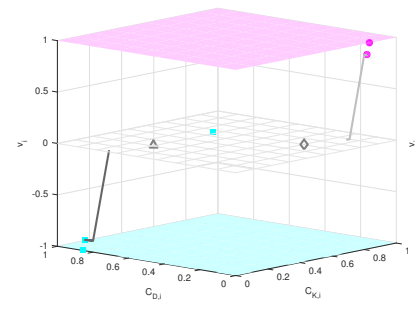

(e) Case 2, $\alpha=0$

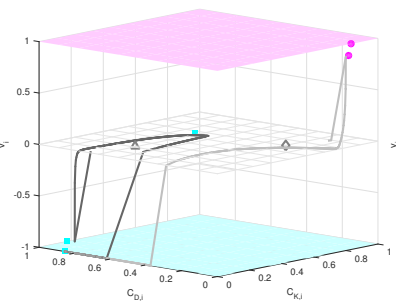

(f) Case $2,10^{-1} \alpha$

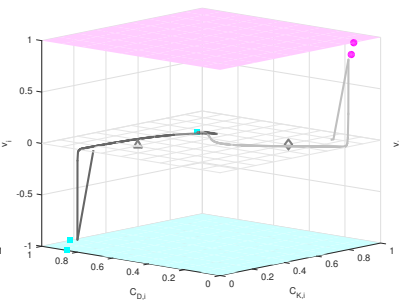

(g) Case 2, $\alpha$

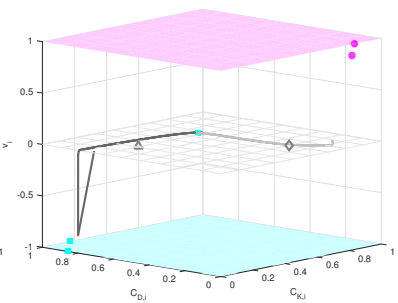

(h) Case 2, $10 \alpha$

FIG. 7. Trajectories of $N=2$ node filaments with $N_{K}=N_{D}=4$ are plotted in the $C_{K, i}-C_{D, i}-v_{i}$ hyperplane in Case 1 with implicitly coordinated nodes (7a-7d) and Case 2 with non-implicitly coordinated nodes (7e-7h). Dark grey (resp. light grey) trajectory represents node 1 (resp. 2). The bottom cyan (top magenta) surface is Regime I (resp. IV) and the mesh is the switching plane between Regime II and III. Stable equilibria with negative (resp. positive) velocities are plotted as cyan squares (resp. magenta dots). The unstable equilibria are grey triangles (resp. diamonds) when in Regime II (resp. III). (7a-7d) Varying $\alpha$ does not affect the direction of motion; however, the coupling improves the transport efficiency. (7e-7h) Node 1 moves retrogradely and node 2 moves anterogradely when decoupled $(\alpha=0)$. Increasing the coupling results in the coordination of nodes. In $7 \mathrm{f}$ the filament moves retrogradely in the limiting regime with $10^{-1} \alpha$. At normal elasticity $\alpha$ and stiffer, the filament exhibits slow motion, the tug-of-war is not resolved (7g and $7 \mathrm{~h})$.

case, the trajectory of one node enters Regime IV in which it is trapped as Regime IV is invariant. The existence of these invariant regimes (I and IV) allows the model to detect the speed-up of transport.

In Figures 7e-7h, nodes are not initially implicitly coordinated. When uncoupled, node 1 reached the negative velocity $v_{I I}^{*}$ whereas node 2 moves eventually anterogradely at the velocity $v_{I I I}^{*}(\alpha=0,7 \mathrm{e})$; however, the elastic coupling induces their coordination. In Figure $7 \mathrm{f}\left(10^{-1} \alpha\right)$, node 1 imposes its dynamics to node 2 and the filament reaches eventually 
the velocity $v_{I}^{*}$. When filaments get stiffer, neither node can dominate, the tug-of-war is unresolved and the filament moves slowly at $v_{\text {Slow }}^{*}(7 \mathrm{~g}$ and $7 \mathrm{~h})$. The strength of coupling not only changes the mode of motion in the long run but also the transient behaviour $(7 \mathrm{~g}$ and $7 \mathrm{~h})$.

Summing up, with the base value for coupling $\alpha$ there is always coordination of motors along a filament. The coordination is driven by the local dynamics governing each node (initial conditions of nodes belong to the same basin of attraction) or by the elastic property of the filaments. While initial conditions are a major determinant of the final motion of filaments, the elastic properties ensure the coordination of motors along the filament and tune the whole dynamics.

\section{B. Impact of the strength of elastic coupling on transport}

To investigate how the strength of elastic coupling influences the dynamics of filaments composed of 2 nodes, we perform 10,000 simulations with initial conditions $C_{K, i}(0)$ and $C_{D, i}(0)$ uniformly distributed on $[0,1]$ for $i \in\{1,2\}$ for different values of coupling ranging from 0 (no coupling) to $10^{3} \alpha$. For comparison purpose, simulations are run for each case with the same initial conditions for all the coupling values; results are provided in Figures 8 to 12 . Figures 8 to 11 focus on the effect of coupling on the long run dynamics by comparing the coupled to uncoupled nodes dynamics whereas Figure 12 compiles changes in the long run dynamics as the strength of coupling increases. Additionally, Figures 8 to 11 specify the effect of coupling on the filament dynamics depending on their initial conditions.

Figures 8a-8h show clouds of coloured circles (as in Figure 6 the colours of circles represent final velocities $v^{*}$ ), representing initial conditions of nodes for Case 1 for the eight strengths of coupling considered. Results for Case 2 to 4 are provided in Figures 9a-9h, 10a-10h and 11a-11h. Figures 8i-8p, 9i-9p, 10i-10p, 11i-11p show the effect of coupling in the regions of implicit coordination; circles only represent the initial conditions where the coupling changes the final velocity of coupled nodes with respect to the uncoupled case. Colour of the inner (resp. outer) circle represents the final velocity of uncoupled (resp. coupled) nodes. Then, in Figures 8q-8x, 9q-9x and 11q-11x we focus on regions in which the motor coordination is only due to elastic coupling (not implicitly coordinated nodes).

In Figures 8 to 11 , for a coupling value from $10^{-3} \alpha$ to $10^{3} \alpha$, only simulations, which have 
at the final time $t_{\text {final }}$ both nodes at the same final velocity $\left(\left|v_{1}\left(t_{\text {final }}\right)-v_{2}\left(t_{\text {final }}\right)\right|<10^{-3}\right)$, are considered. With $t_{\text {final }}=60 \mathrm{~s}$, less than $1 \%$ of simulations do not converge to steady states (do not reach the coordination of motors) except for Case 1 with $10^{-3} \alpha(8 \mathrm{~b})$. Note that the as yet unstabilized simulations in Figure 8b have initial conditions in the regions of non-implicit coordination. However, when these cases are run for a longer $t_{\text {final }}=180 \mathrm{~s}$, all simulations reach steady states (15b). With $\alpha>0$, the coordination of nodes is always observed even if in some cases, such as for strong motors considered with soft coupling, it takes longer to reach steady states.

Figure 12 summarizes data shown in Figures 8-11 by representing the statistical features of filament motion as functions of the coupling strength. The proportion of filaments reaching one of the final velocities $\left(v_{I}^{*}, v_{I I}^{*}, v_{S l o w}^{*}, v_{I I I}^{*}\right.$ and $\left.v_{I V}^{*}\right)$ is indicated by the size of the dot shown in each condition. The changes in filament final velocity $v^{*}$ observed as the elastic coupling increases are indicated by a line, whose width represents the proportion of filaments undergoing this change.

Filaments can reach five final velocities (Figure 12): the maximal velocity $v_{I}^{*}=-v_{f D}$ (resp. $v_{I V}^{*}=v_{f K}$ ) corresponding to the unique GAS equilibrium in Regime I (resp. IV), $v_{I I}^{*}$ (resp. $v_{I I I}^{*}$ ) corresponding to the LAS equilibrium in Regime II (resp. III) and another LAS equilibrium in Regime II or III with velocity $v_{\text {Slow }}^{*}$. When motors are strong (Case 1 or 3), most filaments move in a dynein-driven motion, in particular if dynein has a catchbond off rate (Case 3). In these cases, the elastic coupling increases the final velocity of implicitly coordinated filaments (12b and $12 \mathrm{~h})$. For the non-implicitly coordinated filaments, increasing the coupling can lead to a complete inversion of the filament motion. When motors are weak (Case 2 and 4), the tug-of-war is rarely resolved and the majority of filaments undergoes a slow motion, in particular if the nodes are implicitly coordinated. However, elastic coupling has an interesting effect when nodes are non-implicitly coordinated (12f and 121). A soft elastic coupling $(<\alpha)$ tends to promote a resolution of the tug-of-war towards the maximal velocity equilibria $\left(v_{I}^{*}\right.$ and $\left.v_{I V}^{*}\right)$; however, this phenomenon is abolished when the elastic coupling increases $(>\alpha)$. The slow motion $v_{S l o w}^{*}$ is only observed for weak motors (Case 2 and 4 ). With mixed rates (Case 3 and 4 ), the maximal anterograde velocity $v_{I V}^{*}$ is not reachable from initial conditions in Regime II or III. 


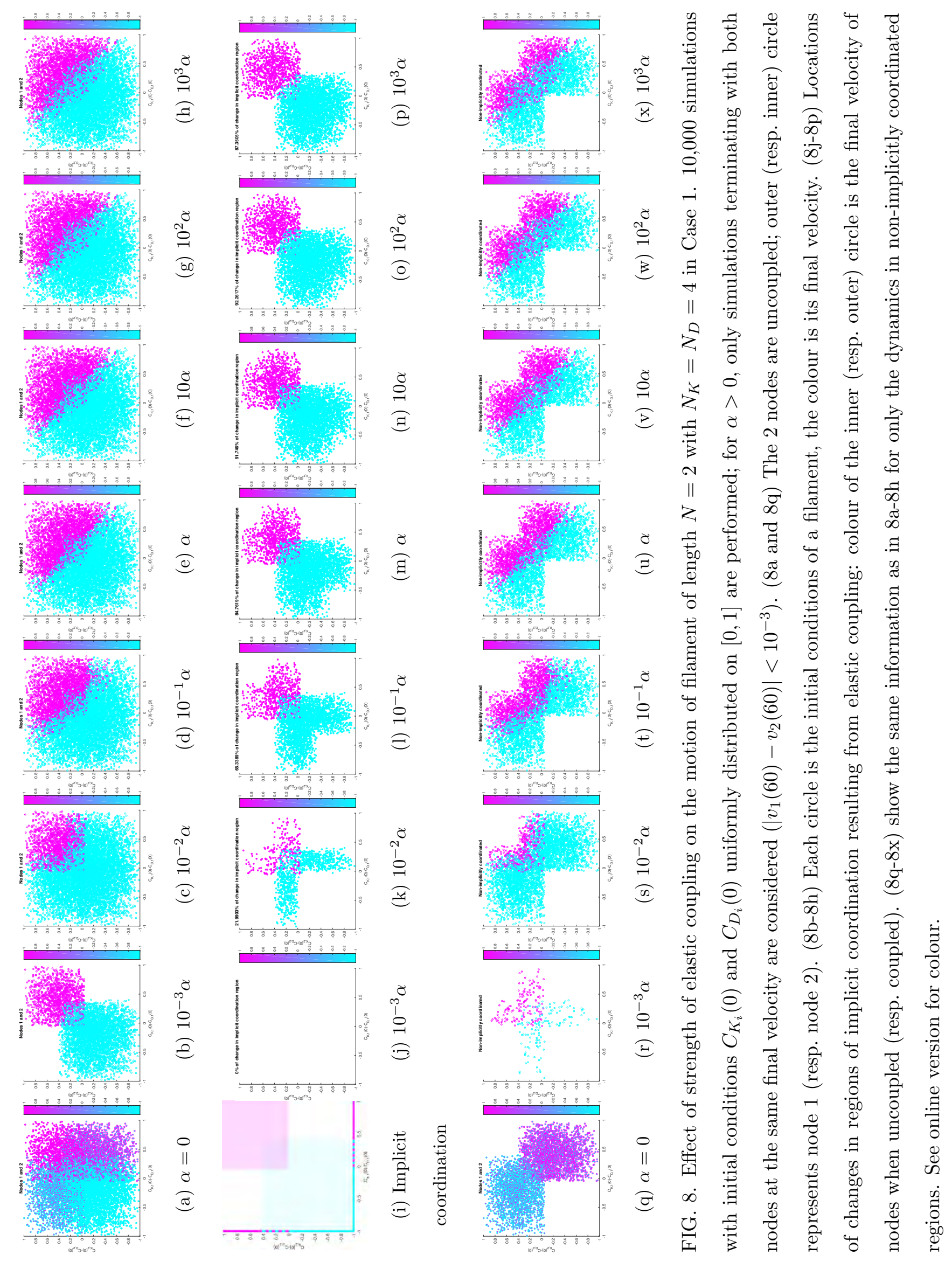




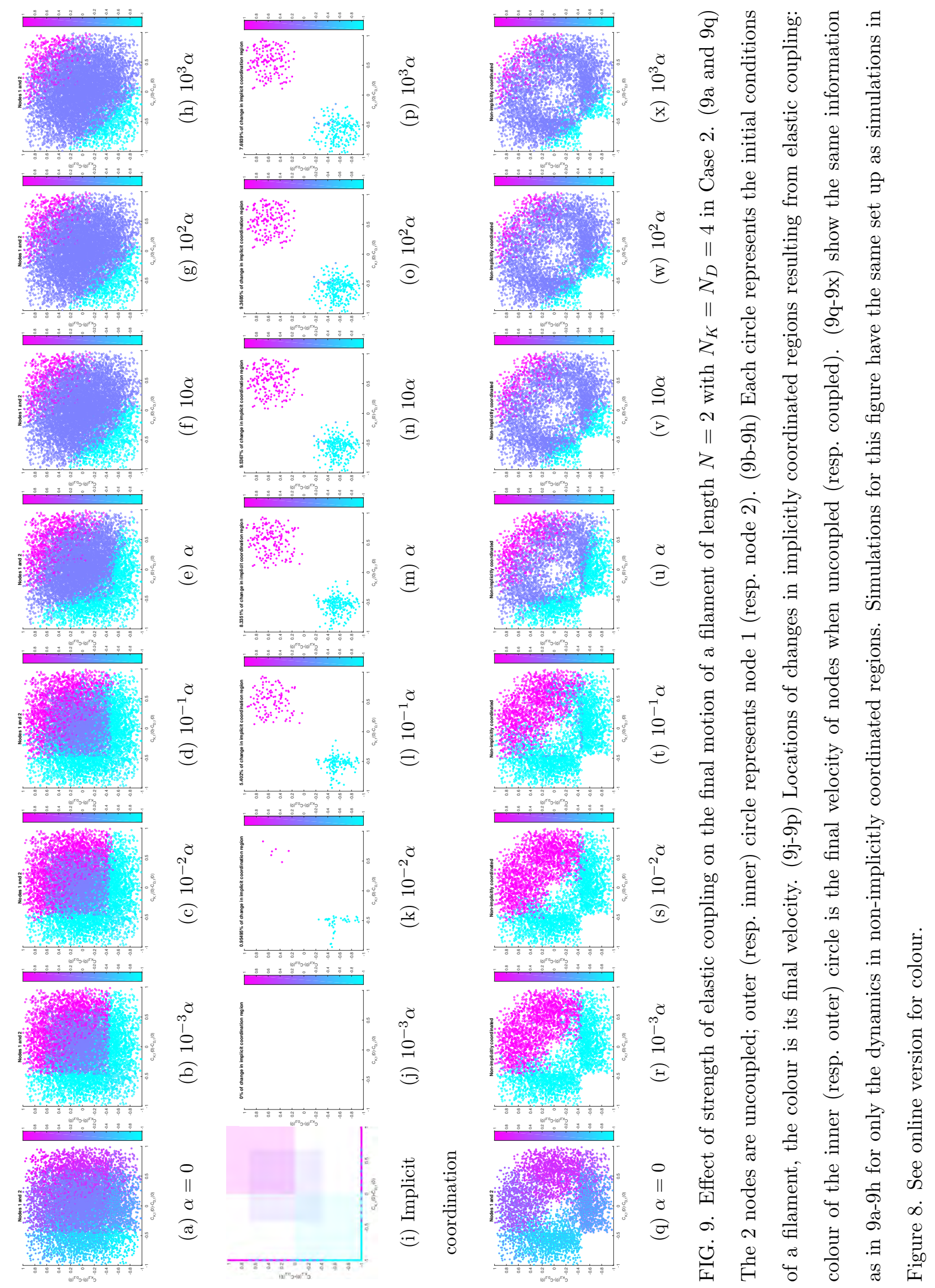




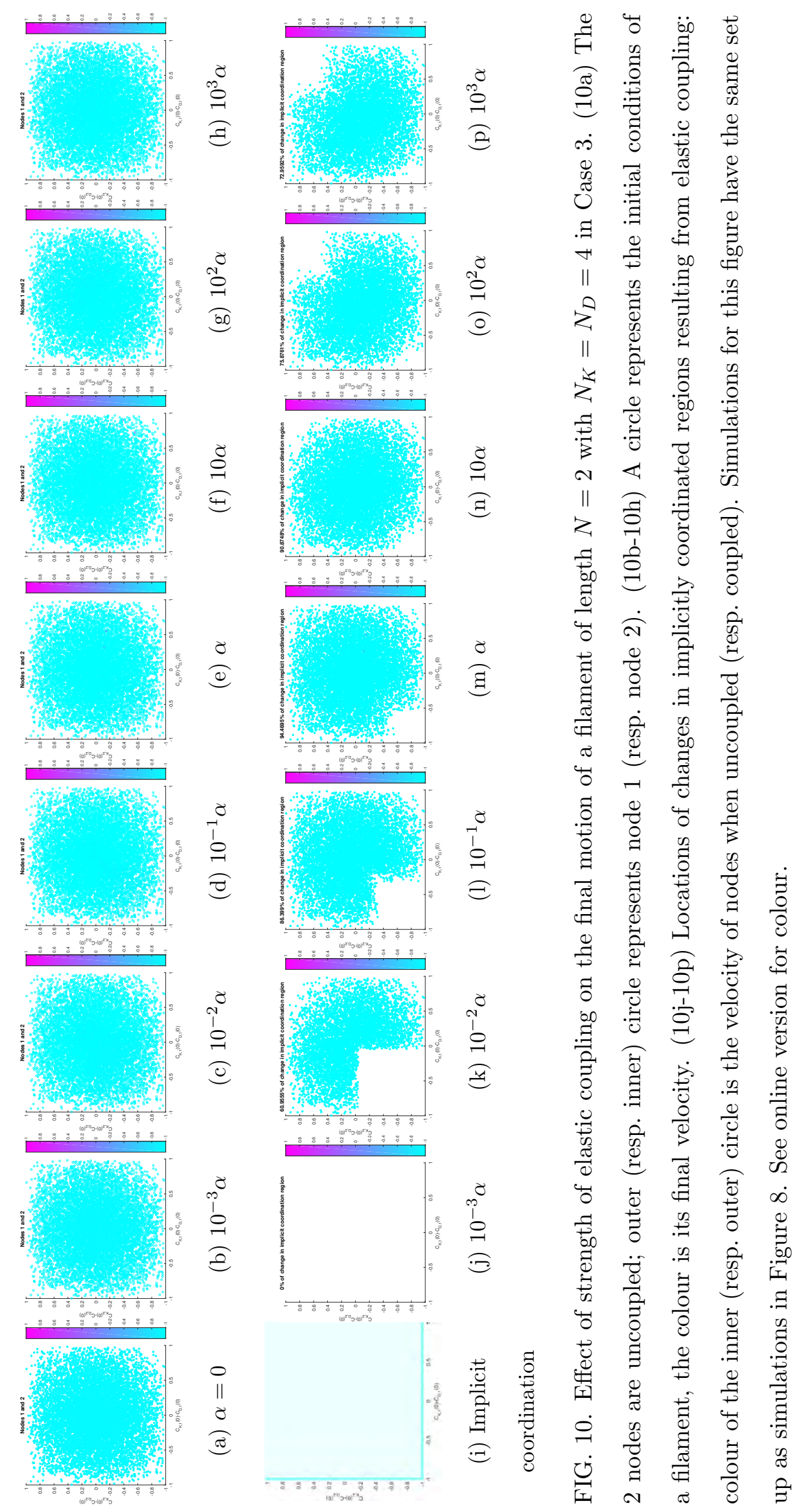




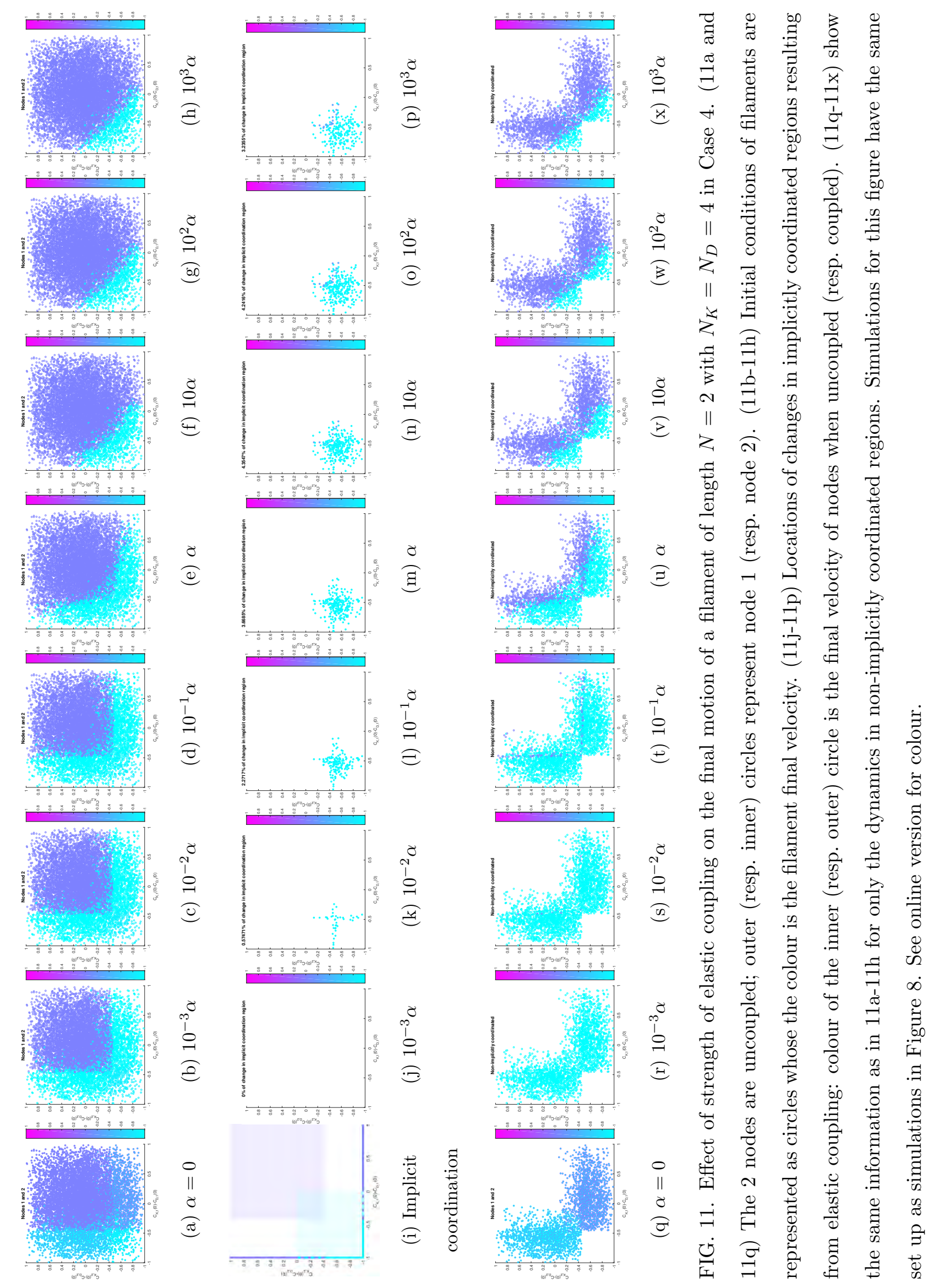




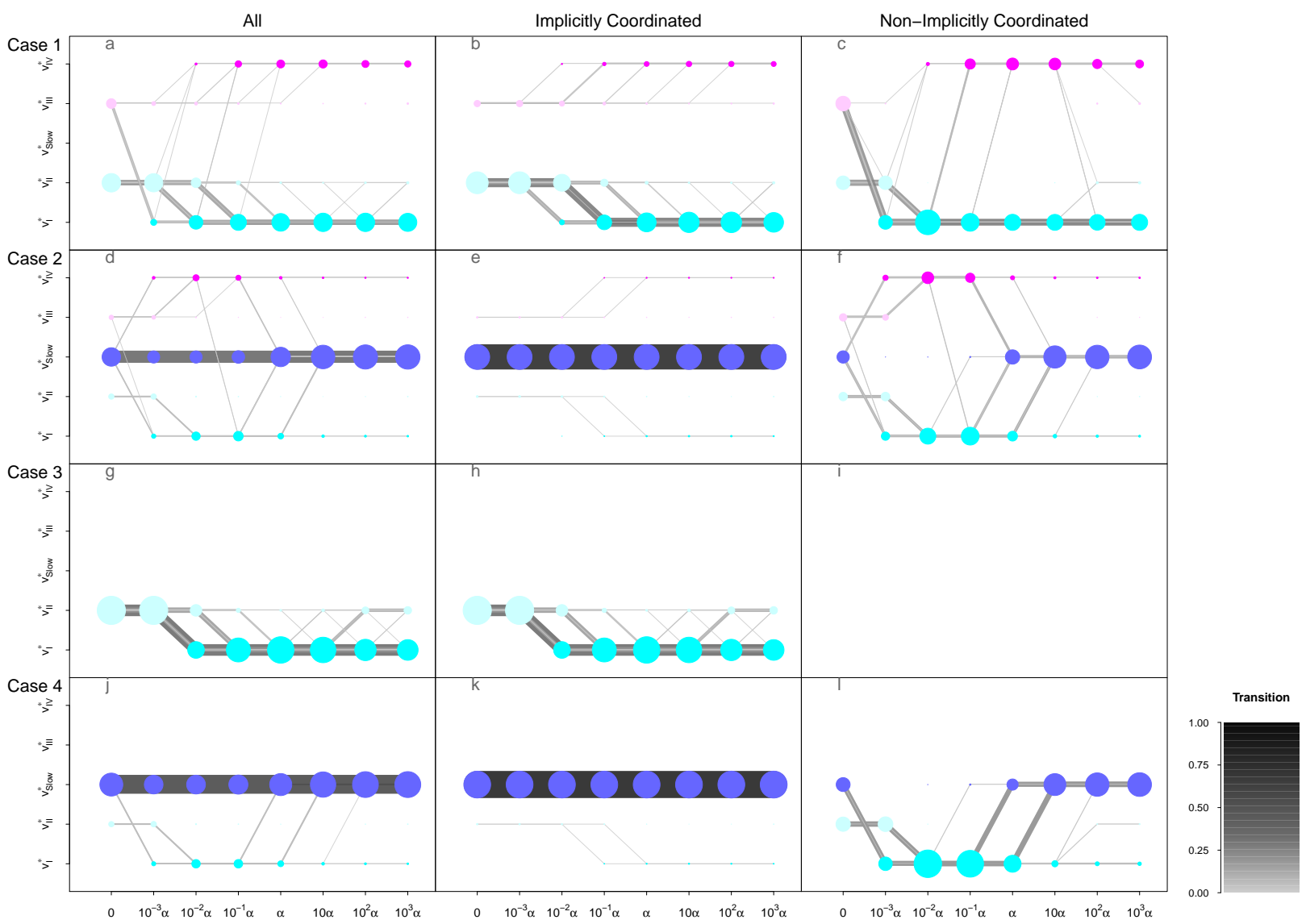

FIG. 12. Impact of the elastic coupling on filament final velocities $v^{*}$. Proportions of nodes $(\alpha=0)$ or filaments reaching a final velocity $\left(v_{I}^{*}, v_{I I}^{*}, v_{S l o w}^{*}, v_{I I I}^{*}\right.$ or $\left.v_{I V}^{*}\right)$ are shown as the coupling increases. Dot size codes for the proportion value. In the colour version, cyan is $v_{I}^{*}$, light cyan $v_{I I}^{*}$, purple blue $v_{\text {Slow }}^{*}$, light magenta $v_{I I I}^{*}$ and magenta $v_{I V}^{*}$. Additionally, the transitions of $v^{*}$ between the possible equilibrium values as the coupling strength increases are shown. Each path shows how the final velocity of a given filament evolves as the coupling increases. The line width and greyscale indicate the proportion of filaments whose final velocity undergoes a specific pattern of transitions between equilibrium values. For readability, only paths with a frequency larger than 0.0075 are shown (if there is a unique transition pattern as the coupling increases, its frequency is 1). Proportions and transitions of $v^{*}$ are given for all filaments in $(\mathrm{a}, \mathrm{d}, \mathrm{g}$ and $\mathrm{j})$, for filaments starting with initial conditions in regions of implicit and non-implicit coordination in (b, e, h and k) and (c, f, i and l) respectively. For non-implicitly coordinated nodes (c, $\mathrm{f}$ and $\mathrm{l}$ ) and $\alpha=0$, results for the node 1 are used; results for node 2 are symmetrical. Data used for (a, d, g and j) are shown in Figures 8-11 panels (a) to (h), for (b, e, h and k) in Figures 8-11 panels (i) to (p) and for (c, f, i and l) in Figures 8,9 and 11 panels (q) to (x). Only simulations satisfying $\left|v_{1}\left(t_{\text {final }}\right)-v_{2}\left(t_{\text {final }}\right)\right|<10^{-3}$ for all positive coupling strengths are considered.2Among the 10,000 simulations, for Case 1, 94.3\% of simulations are considered, $99.6 \%$ for Case $2,95.6 \%$ for Case 3 and $99.8 \%$ for Case 4 . 


\section{Elastic coupling increases the final velocity of fast moving filaments}

For uncoupled nodes $(\alpha=0$ in $12 \mathrm{a}, 12 \mathrm{~d}, 12 \mathrm{~g}$ and $12 \mathrm{j})$, the final velocities are the equilibrium values $v_{I I}^{*}$ and $v_{I I I}^{*}$ of Regime II or III but never reach the maximal velocities $v_{I}^{*}$ and $v_{I V}^{*}$ of Regime I and IV. In contrast, coupled nodes reach the maximal velocities of Regime I and IV $\left(10^{-3} \alpha-10^{3} \alpha\right.$ in $12 \mathrm{a}, 12 \mathrm{~d}, 12 \mathrm{~g}$ and $\left.12 \mathrm{j}\right)$.

For strong motors (Case 1 and 3, 12a and 12g), as the strength of elastic coupling increases, the majority of filaments run in limiting regimes. In particular for Case 1, when the elastic coupling is solely responsible for node coordination, i.e., for filaments starting with non-implicitly coordinated nodes $(12 \mathrm{c})$, most filaments move at maximal velocities $v_{I}^{*}$ and $v_{I V}^{*}$

In regions of implicit coordination, the long run dynamics is unchanged by a soft coupling $10^{-3} \alpha(8 \mathrm{j}, 9 \mathrm{j}, 10 \mathrm{j}, 11 \mathrm{j}, 15 \mathrm{~d}$ and $12 \mathrm{~b}, \mathrm{e}, \mathrm{h}, \mathrm{k})$, whereas a coupling $\geq 10^{-2} \alpha$ can affect the final velocity and accelerate the transport (8k-8p, 9k-9p, 10k-10p, 11k-11p and 12b,e,h,k).

For strong motors, in Case 1, when nodes are implicitly coordinated, stronger coupling causes more filaments to end up in the limiting regimes $(8 \mathrm{k}-8 \mathrm{p}) .22 \%$ of filaments are speed up from Regime II (when uncoupled) to I or III (when uncoupled) to IV with a coupling of $10^{-2} \alpha(8 \mathrm{k})$, whereas, with $10 \alpha$ or $10^{2} \alpha$, about $92 \%$ of filaments are accelerated by the coupling (8n-8o). This phenomenon is captured by the transitions of final velocities from Regime II (resp. III) to Regime I (resp. IV) equilibria as the coupling increases (12b). Note that the coupling does not induce any change in the dynamics when one of the motors strongly dominates (no circle in bottom left and top right corners in 8m). In Case 3 where all nodes are implicitly coordinated by initial conditions, the base value $\alpha$ optimizes the transport efficiency: there is the largest proportion of filaments running in the limiting Regime I (12g-h) and about $95 \%$ of the final dynamics are accelerated with respect to the uncoupled case $(10 \mathrm{~m})$. For coupling between $10^{-2} \alpha$ and $10^{3} \alpha$, at least $60 \%$ of filaments run faster $(10 \mathrm{k}-10 \mathrm{p})$. Transitions in $12 \mathrm{~h}$ show that increasing the coupling up to $10^{2} \alpha$ might accelerate the transport; however, some filaments are slowed down to $v_{I I}^{*}$ once the coupling gets larger than the base value $\alpha$.

For weak motors, when nodes are implicit coordinated, the coupling has less effects; at most, less than 10\% in Case $2(9 \mathrm{k}-9 \mathrm{p})$ and $5 \%$ in Case $4(11 \mathrm{k}-11 \mathrm{p})$ of filaments are accelerated by the coupling. However, for weak motors, when implicitly coordinated, filaments are 
mostly in slow motions as shown in Figures 12e and 12k.

Hence, we can conclude that the coupling promotes the motion of filaments in limiting regimes when the tug-of-war is resolved.

\section{Soft coupling helps the resolution of tug-of-war}

With weak motors (Case 2 and 4 ), large proportion of filaments moves in a slow motion $v_{\text {Slow }}^{*}(12 \mathrm{~d}$ and $12 \mathrm{j})$ indicating that there is no winner to the competition (kinesins and dyneins stay attached to the nodes); the tug-of-war is not resolved. The lowest proportions of filaments under slow motion are obtained with $10^{-3} \alpha-10^{-1} \alpha(12 \mathrm{~d}$ and $12 \mathrm{j})$. Soft couplings help the resolution of tug-of-war.

In regions of implicit coordination, the coupling does not help the resolution of tug-ofwar $(12 \mathrm{e}$ and $12 \mathrm{k})$. In $9 \mathrm{j}-9 \mathrm{p}$ and $11 \mathrm{j}-11 \mathrm{p}$, there is no occurrence of purple blue inner circles combined with cyan or magenta outer circles at any strength of coupling. However, in regions where the coordination is solely due to coupling, a soft coupling $\left(10^{-3} \alpha-10^{-1} \alpha\right)$ promotes the resolution of tug-of-war (12f, 12l, 9r-9t and 11r-11t). With $10^{-3} \alpha$, no filaments with non-implicitly coordinated nodes run in slow motion and less than $1 \%$ of filaments with $10^{-1} \alpha$ (12f, 12l, 9r-9t and 11r-11t). Contrarily, increasing the strength of coupling beyond the base value $\alpha$ is unfavourable to the resolution of tug-of-war (9r-9x, 11r-11x, 12f and 12l). Stiffer filaments $(\geq 10 \alpha)$ are mostly stalled in a slow motion, weak motors do not manage to resolve the tug-of-war when nodes are non-implicitly coordinated $(12 \mathrm{f}, 12 \mathrm{l}, 9 \mathrm{v}-9 \mathrm{x}$ and $11 \mathrm{v}-11 \mathrm{x})$.

Generally, with weak motors, soft coupling helps the resolution of tug-of-war whereas stiff filaments $(\geq 10 \alpha)$ mostly move under slow motions (12d and $12 \mathrm{j})$.

\section{Strength of coupling changes the mode of motion}

For Case 1 (strong motors considered with exponential off rates), when the coupling is solely responsible for motor coordination, increasing the strength of coupling favours the anterograde motion $(8 \mathrm{r}-8 \mathrm{x}, 15 \mathrm{f}$ and see transitions in $12 \mathrm{c})$. Note that the base value $\alpha$ provides the largest proportions of filament moving at $v_{I V}^{*}(12 \mathrm{c})$. In addition, with a coupling $\geq \alpha$ and in limited regions of implicit coordination where there is the balance between kinesin 
and dynein binding site occupancy at one node (cyan inner circles combined with magenta outer circles in $8 \mathrm{~m}-8 \mathrm{p}$ ), the coupling changes the motion from retrograde to anterograde; the coupling helps kinesin in these rare cases (those transitions do not appear in $12 \mathrm{c}$ as their frequencies are below 0.0075). Furthermore, the increase of the coupling strength beyond $10 \alpha$ can change the motion from anterograde to retrograde or slow down the speed of the anterograde transport (12c).

Finally, with weak motors (Case 2 and 4 ), for stiff filaments, $\geq 10 \alpha$, in very particular zones of implicit coordination regions, we can observe from 5 to 20 cyan inner circles combined with purple blue outer circles. In these rare cases (those transitions do not appear in 12c as their frequencies are below 0.0075), the coupling of two initially driven by dynein nodes results in the stalling of motion and an increase of kinesin loading (9n-9p and 11n-11p). As previously mentioned, with weak motors, for non-implicitly coordinated filaments, soft coupling promotes the motion in limiting regimes whereas coupling $\geq \alpha$ stalls filaments under slow motions (12f and 12l). Remark that in Case 2, increasing the coupling from $10^{-2} \alpha$ to $10^{-1} \alpha$ changes the motion of some filaments from anterograde to retrograde transport (12f).

Summing up, the elastic coupling always facilitates the coordination of motors along filaments and optimizes the transport efficiency by promoting the motion of filaments in limiting Regime I and IV. Above $10^{-2} \alpha$, when the initial conditions define the mode of motion (regions of implicit coordination), the coupling tunes the dynamics by accelerating the speeds of fast filaments. However, when filaments are under slow motion (the tug-of-war is not resolved), the coupling has no effect. Hence, the coupling affects more filaments when motors are strong. Soft filaments $\left(\leq 10^{-3} \alpha\right)$ take longer to converge to steady states (maximal velocities) when the coupling is solely responsible for the coordination of motors; and, when initial conditions dominate, the coupling does not change the final dynamics and filaments remain in intermediate Regime II and III. Moreover, when coupling is solely responsible for motor coordination, the strength of coupling can also affect the mode of motion. With strong motors, any coupling $\geq 10^{-3} \alpha$ promotes motion in limiting regimes whereas, with weak motors, only soft couplings $(<\alpha)$ boosts fast motions and stiffer filaments move in slow motions. 


\section{Conclusion}

A deterministic model for the transport of elastic filaments by antagonistic motor proteins is derived following a mean field approach and assuming a local tug-of-war between motors at nodes coupled by springs. The assumption that motors share the load equally is justified by the low density of motors per unit length of filaments allowed in the model. The model is developed to unravel possible mechanisms of the intracellular transport of intermediate filaments by kinesin and dynein along microtubules in cells but may be applied to the motion of other elastic elongated structures such as mitochondria. Since motor properties are still under debate we investigate two combinations of force-dependent off rates with two motor strengths. This also allows us to reveal filament behaviour specific to a unique combination of motor properties. The theoretical approach is initially tuned to accommodate mechanical properties of intermediate filaments. The base value of the spring constant $\alpha$ is estimated from the range of Young's modulus of intermediate filaments (see e.g. $[4,7]$ ). Then, a range of values for the strength of coupling based on the value $\alpha$ is considered to theoretically assess the impact of the elastic properties on filament motion. The mathematical model is a $3 N$-dimensional system of differential equations defined over 4 different regimes. Due to the high dimension and number of parameters of the model, the exhaustive characterization of the very rich dynamics of the model is challenging. We have characterized equilibrium points and their stability: up to 7 equilibrium points ( 5 in intermediate Regime II and III and 1 in each limiting Regime I and IV) can exist. Their local stability conditions have been determined analytically. Finally, their basin of attraction (for locally asymptotically stable equilibrium) have been numerically investigated. Note that the dynamics of uncoupled nodes with exponential off rates for motors $(\alpha=0)$ follows the results of [18].

When both exponential off rates are considered, initial conditions and parameter values can be found to result in three types of motion (fast retrograde, slow motion, fast anterograde). For mixed rates, only two types of transport are observed (fast retrograde and slow motion). The slow motion velocities $v_{\text {Slow }}^{*}$ can be positive. For the parameter values used in this theoretical study, the use of catch-bond detachment rate for dynein does not allow us to reproduce the experimental observations of fast anterograde transport of intermediate

filaments [14]. However, for other parameter values, the small positive values of $v_{\text {Slow }}^{*}$ might be significantly larger. Note that only slow filaments are observed when dynein are weak 
$\left|F_{s D}\right| / F_{d D}<1.1$ with both rate combinations.

Overall, initial conditions and the strength of elastic coupling dictate the final motion of filaments. Most importantly, we show that a global coordination of motors along a filament emerges from the tuning of local dynamics at the nodes by elastic coupling. When uncoupled nodes move in the same direction due to their initial conditions (implicitly coordinated nodes), the elastic coupling rarely changes the direction of motion but can alter their velocity. Hence, the intracellular context, which defines the initial conditions for the system, is essential in determining the mode of transport of filaments. In addition, the elasticity of filaments plays a major role in their motility by ensuring the coordination of motors making the system more robust. More interestingly, the elastic coupling optimizes the transport efficiency by promoting the motion at maximal velocities in limiting regimes I and IV (Figure 13). The coupling has a more pronounced effect when motors are strong (13a and 13c). When the coupling is solely responsible for the motor coordination, the strength of coupling affects the efficiency of motors and changes the dynamics. For strong motors, the strength of coupling speeds up the motor coordination. When motors are weak, the tug-of-war is rarely resolved and the majority of filaments undergoes a slow motion (13b and 13d), in particular when the motor coordination is due to initial conditions. However, elastic coupling has an interesting effect when it is solely responsible for the motor coordination. A soft elastic coupling $(<\alpha)$ helps the resolution of the tug-of-war towards the maximal velocities ( $v_{I}^{*}$ and $\left.v_{I V}^{*}\right)$; however, this phenomenon is overturned for stiffer filaments $(>\alpha)$.

The deterministic model considered here is derived from a stochastic model developed in [22] to study the same biological problem, the transport of intermediate filaments by antagonistic motors along a microtubule. This stochastic model describes the position of nodes $x_{i}$ in 1-dimension (along the microtubule) and the numbers of motors attached to nodes $n_{\bullet}, i$. The node positions $x_{i}$ satisfy a system of differential equations obtained by considering (1) with $v_{i}=\frac{d x_{i}}{d t}$ and the numbers of attached motors are governed by stochastic processes, simulated by Gillespie's algorithm, that determine the effective binding $\left(N_{\bullet}-n_{\bullet}, i\right) \pi_{\bullet}$ and detachment $n_{i, \boldsymbol{\bullet}} \epsilon_{\bullet} \exp \left[\frac{\mid F_{\bullet}, i}{F_{d \bullet}}\right]$ rates of motors at nodes. In this stochastic model the node positions and on and off rates are functions of forces $F_{\bullet}, i$ acting on nodes. This stochastic formulation helps us to capture the transient behaviour of filaments, such as direction switching, and the dynamics of nodes within a filament, such as "traveling waves of coordination of motors" along a filament [22]. The stochastic model investigates the effect of the filament 


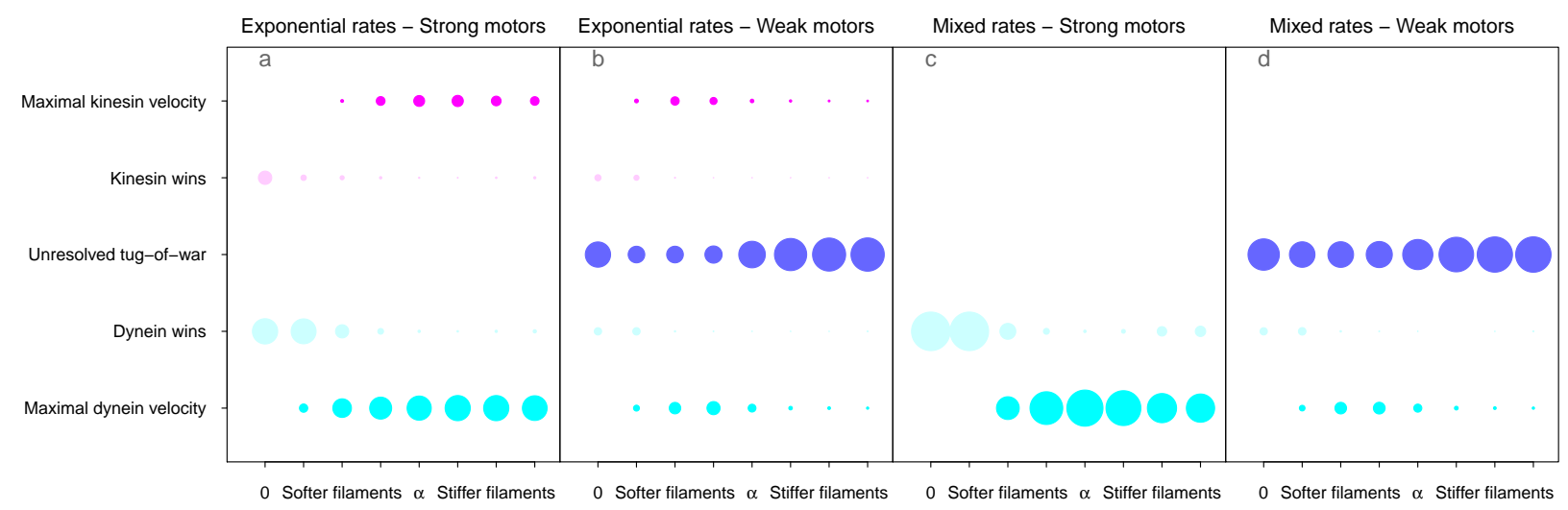

FIG. 13. Summary of the impact of the elastic coupling on filament motion. Proportions of nodes $(\alpha=0)$ or filaments reaching a specific mode of motion are shown as the elastic coupling strength increases. The possible modes of motion reached by filaments are: (Maximal dynein velocity) (cyan) filaments are transported retrogradely at the maximal dynein velocity, (Dynein wins) (light cyan) filaments are transported retrogradely, (Unresolved tug-of-war) (purple blue) filaments are stalled in a slow retrograde or anterograde motion, (Kinesin wins) (light magenta) filaments are transported anterogradely and (Maximal kinesin velocity) (magenta) filaments are transported anterogradely at the maximal kinesin velocity. Dot sizes represent proportions of filaments in different modes of motion. See online version for colour.

properties such as the number of motor binding sites, length and elasticity on filament motion. However, with this formulation, the characterization of steady modes of motion (i.e. transport of filaments at steady velocities) is difficult. Hence, following steps described in Appendix B, we derive the expression (B18) for the dynamics of the node velocity $v_{i}$ and express forces $F_{\bullet}, i$ acting on motors as functions of $v_{i}$. Finally, by combining the on and off rates expressed as functions of $v_{i}$, a system of differential equations for the dynamics of binding sites occupied by motors is coupled to the dynamics of $v_{i}$. Hence, we obtain this deterministic model that allows a steady state analysis. Conclusions of both the stochastic and deterministic models in the long run are in agreement. Furthermore, the deterministic model is defined over 4 regimes following the force-velocity relationships for motor molecules (Figure 14). The limiting Regime I and IV result from the model assumption that there is a maximum forward velocity for both motor molecules. Although this is a reasonable assumption, the trapping behaviour in Regime I and IV (due to the invariance under the 
flow of (2) and (3)) is likely an unrealistic artifact of the model. However, the presence of these invariant regimes has given insights into the system, such as the detection of the speed-up of the system, which would not have been possible without the trapping behaviour. Although the dynamics of the biological system would not be trapped, we believe the maximum velocities would be attracted to or fluctuate around a small range of velocities near the trapping velocities reported here.

Finally, in this work we do not investigate the effects of biochemical properties of intermediate filaments (number of binding sites and desorption constants) that post-translational modifications, bundling or binding of associated proteins might modify. This will be a subject of further investigations.

\section{Appendix A: Force-Velocity relationship for single motors}

Let $F_{s K}$ be the stall force of kinesin which is negative. Then the velocity function for kinesin is given by

$$
v_{K}\left(F_{K}\right)=\left\{\begin{array}{lc}
v_{b K}\left(1-F_{K} / F_{s K}\right) & F_{K} \leq F_{s K} \\
v_{f K}\left(1-F_{K} / F_{s K}\right) & F_{s K}<F_{K}<0 \\
v_{f K} & 0 \leq F_{K}
\end{array}\right.
$$

where $v_{f K}$ and $v_{b K}$ are positive constants giving the forward and backward speed of kinesin. Let the stall force for dynein be $F_{s D}$ which is positive. The velocity function for dynein is

$$
v_{D}\left(F_{D}\right)=\left\{\begin{array}{lc}
-v_{f D} & F_{D} \leq 0 \\
-v_{f D}\left(1-F_{D} / F_{s D}\right) & 0<F_{D}<F_{s D} \\
-v_{b D}\left(1-F_{D} / F_{s D}\right) & F_{s D} \leq F_{D}
\end{array}\right.
$$

where $v_{f D}$ and $v_{b D}$ are positive constant representing the forward and backward speed of dynein. These force-velocity relationships are plotted in Figure 14; they are usually used for processive motors; see, for instance, [24].

\section{Appendix B: Model derivation}

Consider all the motor molecules associated with node $i$, from Eq. (1), the force balance equation is $n_{K, i} F_{K, i}+n_{D, i} F_{D, i}+F_{r e s, i}-\mu v_{i}=0$. The maximum force generated by each 


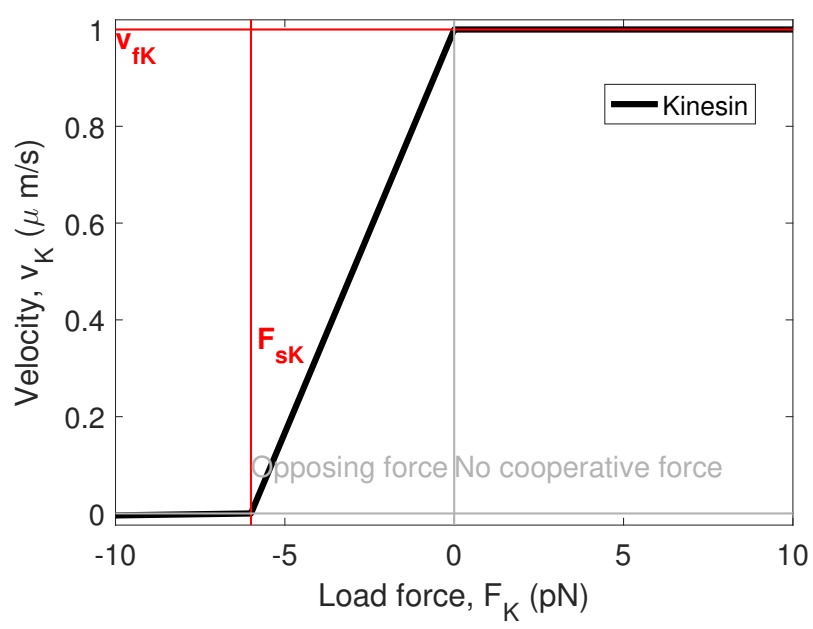

(a) $v_{K}\left(F_{K}\right)$

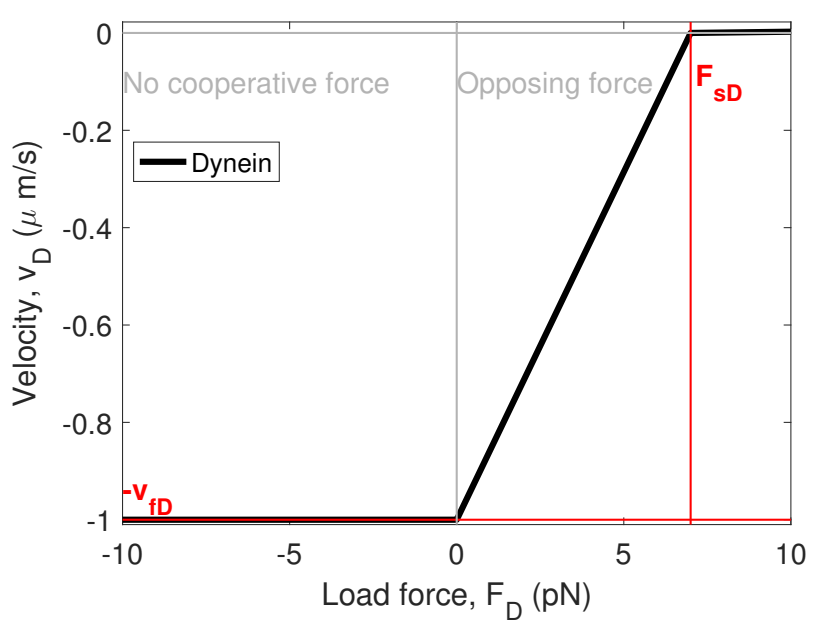

(b) $v_{D}\left(F_{D}\right)$

FIG. 14. Velocity of kinesin and dynein as a function of load force, Eq. (A1) and Eq. (A2).

kinesin molecule is $-F_{s K}\left(\right.$ as $\left.F_{s K}<0\right)$ and similarly the maximum force generated by each dynein molecule is $-F_{s D}$. If $-n_{K, i} F_{s K}-n_{D, i} F_{s D}+F_{r e s, i} \geq 0$, the node velocity $v_{i}$ is positive; kinesin will win the tug-of-war and the node will move to the right. On the other hand, if $-n_{K, i} F_{s K}-n_{D, i} F_{s D}+F_{\text {res }, i} \leq 0$, the node velocity $v_{i}$ is negative; dynein will win the tugof-war and the node will move to the left. When $F_{r e s, i}-n_{D, i} F_{s D}>0$ then the force on the kinesin molecules will be positive (when the load is positive, the kinesin may oppose the load to adjust the velocity to the no-load velocity of kinesin $\left.v_{f K}\right)$. Likewise if $F_{r e s, i}-n_{K, i} F_{s K}<0$ then the force on the dynein will be negative (when the load is negative, the dynein may oppose the load to adjust the velocity to the no-load velocity of dynein $-v_{f D}$ ).

The motor molecules adjust the force to maintain the velocity relation. By equating the piecewise velocities of motors defined in equations (A1) and (A2) and the force balance equation, the velocity $v_{i}$ for node $i$ and the force acting on a kinesin (resp. dynein) attached to node $i F_{K, i}$ (resp. $\left.F_{D, i}\right)$ are obtained as piecewise functions defined over four intervals as follows

$$
v_{i}=\left[\begin{array}{ccc}
-v_{f D} & F_{r e s, i}<n_{K, i} F_{s K} & \text { Dyneins win } \\
\frac{n_{K, i} F_{s K}+n_{D, i} F_{s D}-F_{r e s, i}}{n_{K, i} F_{s K} / v_{b K}-n_{D, i} F_{s D} / v_{f D}-\mu} & n_{K, i} F_{s K} \leq F_{r e s, i}<n_{K, i} F_{s K}+n_{D, i} F_{s D} & \text { Dyneins win } \\
\frac{n_{K, i} F_{s K}+n_{D, i} F_{s D}-F_{r e s, i}}{n_{K, i} F_{s K} / v_{f K}-n_{D, i} F_{s D} / v_{b D}-\mu} & n_{K, i} F_{s K}+n_{D, i} F_{s D} \leq F_{r e s, i} \leq n_{D, i} F_{s D} & \text { Kinesins win } \\
v_{f K} & n_{D, i} F_{s D}<F_{r e s, i} & \text { Kinesins win }
\end{array}\right.
$$


and

$$
F_{K, i}=\left[\begin{array}{cc}
F_{s K}\left(1+v_{f D} / v_{b K}\right) & F_{r e s, i}<n_{K, i} F_{s K} \\
\frac{F_{s K}\left(-n_{D, i} F_{s D}\left(1 / v_{b K}+1 / v_{f D}\right)+F_{r e s, i} / v_{b K}-\mu\right)}{n_{K, i} F_{s K} / v_{b K}-n_{D, i} F_{s D} / v_{f D}-\mu} & n_{K, i} F_{s K} \leq F_{r e s, i}<n_{K, i} F_{s K}+n_{D, i} F_{s D} \\
\frac{F_{s K}\left(-n_{D, i} F_{s D}\left(1 / v_{b D}+1 / v_{f K}\right)+F_{r e s, i} / v_{f K}-\mu\right)}{n_{K, i} F_{s K} / v_{f K}-n_{D, i} F_{s D} / v_{b D}-\mu} & n_{K, i} F_{s K}+n_{D, i} F_{s D} \leq F_{r e s, i} \leq n_{D, i} F_{s D} \\
\frac{-n_{D, i} F_{s D}\left(v_{f K} / v_{b D}+1\right)+F_{r e s, i}-\mu v_{f K}}{n_{K, i}} & n_{D, i} F_{s D}<F_{r e s, i}
\end{array}\right.
$$

and the force acting on one dynein attached to node $i$ is

$$
F_{D, i}=\left[\begin{array}{cc}
\frac{n_{K, i} F_{s K}\left(v_{f D} / v_{b K}+1\right)-F_{r e s, i}-v_{f D} \mu}{-n_{D, i}} & F_{r e s, i}<n_{K, i} F_{s K} \\
\frac{F_{s D}\left(n_{K, i} F_{s K}\left(1 / v_{b K}+1 / v_{f D}\right)-F_{r e s, i} / v_{f D}-\mu\right)}{n_{K, i} F_{s K} / v_{b K}-n_{D, i} F_{s D} / v_{f D}-\mu} & n_{K, i} F_{s K} \leq F_{r e s, i}<n_{K, i} F_{s K}+n_{D, i} F_{s D} \\
\frac{F_{s D}\left(n_{K, i} F_{s K}\left(1 / v_{b D}+1 / v_{f K}\right)-F_{r e s, i} / v_{b D}-\mu\right)}{n_{K, i} F_{s K} / v_{f K}-n_{D, i} F_{s D} / v_{b D}-\mu} & n_{K, i} F_{s K}+n_{D, i} F_{s D} \leq F_{r e s, i} \leq n_{D, i} F_{s D} \\
F_{s D}\left(1+v_{f K} / v_{b D}\right) & n_{D, i} F_{s D}<F_{r e s, i} .
\end{array}\right.
$$

Hence, in the aggregated form, the equation of motion (1) for node $i$ is expressed as:

$$
v_{i}=\frac{a_{K, i} n_{K, i} F_{s K}+a_{D, i} n_{D, i} F_{s D}-a_{K, i} a_{D, i} F_{r e s, i}}{a_{K, i} n_{K, i} F_{s K} / v_{0 K, i}-a_{D, i} n_{D, i} F_{s D} / v_{0 D, i}-a_{K, i} a_{D, i} \mu} .
$$

and the aggregated forms of (B2) and (B3) are

$$
F_{K, i}=\frac{F_{s K}\left(-n_{D, i} F_{s D}\left(1 / v_{0 K, i}+1 / v_{0 D, i}\right)+a_{K, i} F_{r e s, i} / v_{0 K, i}-a_{K, i} \mu\right)}{a_{K, i} n_{K, i} F_{s K} / v_{0 K, i}-a_{D, i} n_{D, i} F_{s D} / v_{0 D, i}-a_{K, i} a_{D, i} \mu}
$$

and

$$
F_{D, i}=\frac{F_{s D}\left(n_{K, i} F_{s K}\left(1 / v_{0 K, i}+1 / v_{0 D, i}\right)-a_{D, i} F_{r e s, i} / v_{0 D, i}-a_{D, i} \mu\right)}{a_{K, i} n_{K, i} F_{s K} / v_{0 K, i}-a_{D, i} n_{D, i} F_{s D} / v_{0 D, i}-a_{K, i} a_{D, i} \mu}
$$

where the parameters for node $i$ are defined as follows

$$
a_{K, i}=\left[\begin{array}{ll}
0 & F_{r e s, i}<n_{K, i} F_{s K} \\
1 & n_{K, i} F_{s K} \leq F_{r e s, i}
\end{array} \quad a_{D, i}=\left[\begin{array}{ll}
0 & n_{D, i} F_{s D}<F_{r e s, i} \\
1 & F_{r e s, i} \leq n_{D, i} F_{s D}
\end{array}\right.\right.
$$

note that since $F_{s K}<0$ and $F_{s D}>0, a_{K, i}$ and $a_{D, i}$ are never both 0 for node $i$. The velocity parameters are

$$
v_{0 K, i}=\left[\begin{array}{cc}
v_{b K} & F_{r e s, i}<n_{K, i} F_{s K}+n_{D, i} F_{s D} \text { Dyneins win } \\
v_{f K} & n_{K, i} F_{s K}+n_{D, i} F_{s D} \leq F_{r e s, i} \text { Kinesins win }
\end{array}\right.
$$




\begin{tabular}{|c|c|c|c|c|}
\hline $\begin{array}{l}\text { Condition } \\
\text { of regime }\end{array}$ & \multicolumn{2}{|c|}{$-v_{f D}$} & $\leq v_{i}<$ & $=v_{i}$ \\
\hline Regime & I & II & III & IV \\
\hline Direction & \multicolumn{2}{|c|}{ Retrograde } & \multicolumn{2}{|c|}{ Anterograde } \\
\hline Speed & Maximal & Intermediate & Intermediate & Maximal \\
\hline$a_{K, i}$ & 0 & 1 & 1 & 1 \\
\hline$a_{D, i}$ & 1 & 1 & 1 & 0 \\
\hline$v_{0 K, i}$ & $v_{b K}$ & $v_{b K}$ & $v_{f K}$ & $v_{f K}$ \\
\hline$v_{0 D, i}$ & $v_{f D}$ & $v_{f D}$ & $v_{b D}$ & $v_{b D}$ \\
\hline
\end{tabular}

TABLE III. Conditions of regime of motion of node $i$, direction, speed and parameter values. Parameter values are given in Table II.

and

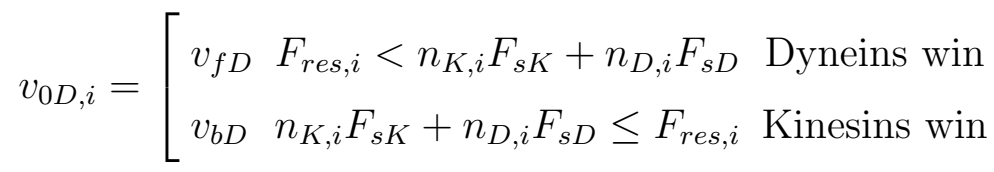

where $v_{f}$ and $v_{b}$ are the forward and backward speeds of either kinesins or dyneins (Table II). Values of $v_{0 \bullet, i}$ and $a_{\bullet, i}$ are also given in Table III.

The formulation of forces (B2)-(B3) acting on motors at node $i$, its velocity (B1) and their aggregated forms (B4)-(B6) were previously developed in [22]; in order to make the content self-contained, they are provided here again.

As previously mentioned, the node velocity (B1) and forces (B2)-(B3) acting on motors at nodes are piecewise functions defined over four intervals (or their aggregated forms (B4)(B6) are defined over four parameter sets (Table III)) determining four different dynamics for the nodes along a filament. These four dynamics are called Regime I, II, III, and IV corresponding to maximal retrograde motion, intermediate retrograde motion, intermediate anterograde motion, and maximal anterograde motion respectively. We want to understand how the nodes along a filament switches between regimes; then, the dynamics of $v_{i}$ is needed. The deterministic extension aiming to access the temporal evolution of node velocities is now described.

First of all, to get the force on the motor molecules as a function of the velocity, $v_{i}$, 
equation (B4) is rewritten as follows

$$
a_{K, i} a_{D, i} F_{r e s, i}=a_{K, i}\left(1-v_{i} / v_{0 K, i}\right) n_{K, i} F_{s K}+a_{D, i}\left(1+v_{i} / v_{0 D, i}\right) n_{D, i} F_{s D}+a_{K, i} a_{D, i} v_{i} \mu
$$

Substituting (B7) in (B5) and (B6) yields :

$$
F_{K, i}\left(v_{i}\right)=\left[\begin{array}{cll}
F_{s K}\left(1-v_{i} / v_{0 K, i}\right) & v_{i}<v_{f K} & \text { Regime I-III } \\
0 & v_{i}=v_{f K} & \text { Regime IV }
\end{array}\right.
$$

and

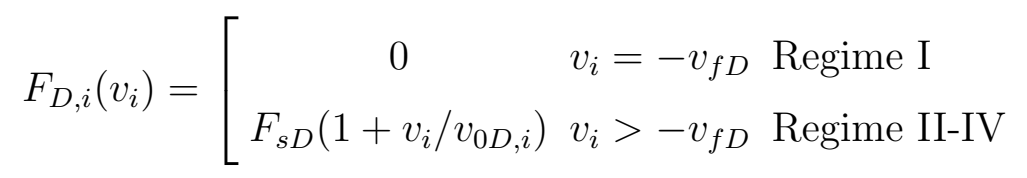

where $v_{i}$ is the velocity of node $i$.

Then, following Kramers's framework, the profiles of force-dependent off rates $f_{\bullet}\left(F_{\bullet}\right)=$ $\exp \left[\frac{\left|F_{\bullet}\right|}{F_{d_{\bullet}}}\right]$ can be expressed as functions of $v_{i}$. The exponential profile for kinesin is

$$
f_{K}\left(F_{K, i}\right)=f_{K}\left(v_{i}\right)=\left[\begin{array}{ccc}
\exp \left[\frac{\left|F_{s K}\left(1+v_{f D} / v_{b K}\right)\right|}{F_{d K}}\right] & v_{i}=-v_{f D} & \text { Regime I } \\
\exp \left[\frac{\left|F_{s K}\left(1-v_{i} / v_{b K}\right)\right|}{F_{d K}}\right] & -v_{f D}<v_{i}<0 & \text { Regime II } \\
\exp \left[\frac{\left|F_{s K}\left(1-v_{i} / v_{f K}\right)\right|}{F_{d K}}\right] & 0 \leq v_{i}<v_{f K} & \text { Regime III } \\
1 & v_{i}=v_{f K} & \text { Regime IV }
\end{array}\right.
$$

and for dynein

$$
f_{D}\left(F_{D, i}\right)=f_{D}\left(v_{i}\right)=\left[\begin{array}{ccc}
1 & v_{i}=-v_{f D} & \text { Regime I } \\
\exp \left[\frac{\left|F_{s D}\left(1+v_{i} / v_{f D}\right)\right|}{F_{d D}}\right] & -v_{f D}<v_{i}<0 & \text { Regime II } \\
\exp \left[\frac{\left|F_{s D}\left(1+v_{i} / v_{b D}\right)\right|}{F_{d D}}\right] & 0 \leq v_{i}<v_{f K} & \text { Regime III } \\
\exp \left[\frac{\left|F_{s D}\left(1+v_{f K} / v_{b D}\right)\right|}{F_{d D}}\right] & v_{i}=v_{f K} & \text { Regime IV } .
\end{array}\right.
$$

The catch-bond profile for dyneins is

$$
f_{D}\left(F_{D, i}\right)=f_{D}\left(v_{i}\right)=\left[\begin{array}{ccc}
1 & v_{i}=-v_{f D} & \text { Regime I } \\
\exp \left[\frac{\left|F_{s D}\left(1+v_{i} / v_{f D}\right)\right|}{F_{d D}}\right] & -v_{f D}<v_{i}<0 & \text { Regime II } \\
\frac{\exp \left(\frac{F_{s D}}{F_{d D}}\right)\left(1-b \exp \left(-\frac{F_{s D}}{F_{d D}}\right)\right)}{\left(1-b \exp \left(\frac{-F_{s D}\left(1+v_{i} / v_{b D}\right)}{F_{d D}}\right)\right)} & 0 \leq v_{i}<v_{f K} & \text { Regime III } \\
\frac{\exp \left(\frac{F_{s D}}{F_{d D}}\right)\left(1-b \exp \left(-\frac{F_{s D}}{F_{d D}}\right)\right)}{\left(1-b \exp \left(\frac{-F_{s D}\left(1+v_{f K} / v_{b D}\right)}{F_{d D}}\right)\right)} & v_{i}=v_{f K} & \text { Regime IV }
\end{array}\right.
$$


where $b=\frac{\exp \left(\frac{F_{s D}}{F_{d D}}\right)}{1.66}$. Different combinations of off rate profiles $f_{\bullet}$ are considered in this work: both exponential off rates for dyneins and kinesins with (B11) and (B10), or catch-bond off rate for dyneins (B12) and exponential profile for kinesins (B10). The latter combination is also called mixed rates.

Finally, to get the dynamics of $v_{i}$, the acceleration of each node can be rewritten by differentiating (B4) expressed with $n_{\bullet}, i=N_{\bullet} C_{\bullet}, i$ as follows:

$$
\frac{d v_{i}}{d t}=\frac{-a_{K, i} a_{D, i} \frac{d F_{r e s, i}}{d t}+\left(1-v_{i} / v_{0 K, i}\right) a_{K, i} \frac{d C_{K, i}}{d t} N_{K} F_{s K}+\left(1+v_{i} / v_{0 D, i}\right) a_{D, i} \frac{d C_{D, i}}{d t} N_{D} F_{s D}}{a_{K, i} C_{K, i} N_{K} F_{s K} / v_{0 K, i}-a_{D, i} C_{D, i} N_{D} F_{s D} / v_{0 D, i}-a_{K, i} a_{D, i} \mu} .
$$

Understanding the dynamics of $v_{i}$ necessitates the definition of the restoring forces. If restoring forces $F_{r e s, i}$ follow Hooke's law, $F_{r e s, i}$ are for tip nodes $i=1$ and $i=N$

$F_{r e s, 1}=-\alpha\left(\left(\left\|\left(x_{1}-x_{2}\right)\right\|-\ell\right) \frac{x_{1}-x_{2}}{\left\|x_{1}-x_{2}\right\|}\right), \quad F_{r e s, N}=-\alpha\left(\left(\left\|\left(x_{N}-x_{N-1}\right)\right\|-\ell\right) \frac{x_{N}-x_{N-1}}{\left\|x_{N}-x_{N-1}\right\|}\right)$

and for interior nodes $i=2, \cdots N-1$

$$
F_{\text {res }, i}=-\alpha\left(\left(\left\|\left(x_{i}-x_{i-1}\right)\right\|-\ell\right) \frac{x_{i}-x_{i-1}}{\left\|x_{i}-x_{i-1}\right\|}+\left(\left\|\left(x_{i}-x_{i+1}\right)\right\|-\ell\right) \frac{x_{i}-x_{i+1}}{\left\|x_{i}-x_{i+1}\right\|}\right)
$$

where $x_{i} \in \mathbb{R}$ is the location of node $i$ in one dimension. The spring constant $\alpha$ is estimated from Young's modulus of intermediate filaments [7] (Table II). Let $\frac{d x_{i}}{d t}=v_{i}$, then

$$
\frac{d F_{r e s, i}}{d t}=-\alpha\left(2 v_{i}-v_{i-1}-v_{i+1}\right)
$$

when node $i$ is an interior node and for the exterior nodes

$$
\frac{d F_{r e s, 1}}{d t}=-\alpha\left(v_{1}-v_{2}\right)
$$

and

$$
\frac{d F_{r e s, N}}{d t}=-\alpha\left(v_{N}-v_{N-1}\right)
$$

Finally, from (B13) and using (B15), the dynamics of $v_{i}$ for an interior node $i$ is described by

$\frac{d v_{i}}{d t}=\frac{a_{K, i} a_{D, i} \alpha\left(2 v_{i}-v_{i-1}-v_{i+1}\right)+\left(1-v_{i} / v_{0 K, i}\right) a_{K, i} \frac{d C_{K, i}}{d t} N_{K} F_{s K}+\left(1+v_{i} / v_{0 D, i}\right) a_{D, i} \frac{d C_{D, i}}{d t} N_{D} F_{s D}}{a_{K, i} C_{K, i} N_{K} F_{s K} / v_{0 K, i}-a_{D, i} C_{D, i} N_{D} F_{s D} / v_{0 D, i}-a_{K, i} a_{D, i} \mu}$.

Model equations for the four regimes are given in the main text as System (2), (3) and (4). 


\section{Appendix C: Dynamics in Regime I and IV}

System (2) and (3) can be both reduced to $N$ planar non-homogeneous linear systems in $C_{K, i}$ and $C_{D, i}$ with bounded solutions in $[0,1] \times[0,1]$. There exists the same unique positive equilibrium for each planar system obtained from $\frac{d C_{K, i}}{d t}=\frac{d C_{D, i}}{d t}=0$, which is

$$
\left(C_{K, i}^{*}, C_{D, i}^{*}\right)= \begin{cases}\left(C_{K I}^{*}, C_{D I}^{*}\right)=\left(\frac{1}{1+\kappa_{K} f_{K}\left(-v_{f D}\right)}, \frac{1}{1+\kappa_{D}}\right), & \text { in Regime I } \\ \left(C_{K I V}^{*}, C_{D I V}^{*}\right)=\left(\frac{1}{1+\kappa_{K}}, \frac{1}{1+\kappa_{D} f_{D}\left(v_{f K}\right)}\right), & \text { in Regime IV }\end{cases}
$$

The matrices of the corresponding planar homogeneous linear systems are

$$
J_{I}=\left[\begin{array}{cc}
-\pi_{K}-\epsilon_{K} f_{K}\left(-v_{f D}\right) & 0 \\
0 & -\pi_{D}-\epsilon_{D}
\end{array}\right], \quad J_{I V}=\left[\begin{array}{cc}
-\pi_{K}-\epsilon_{K} & 0 \\
0 & -\pi_{D}-\epsilon_{D} f_{D}\left(v_{f K}\right)
\end{array}\right] .
$$

Recall that all off rate profiles satisfy $f_{\bullet}(v)>0$ for $v \in\left[-v_{f D}, v_{f K}\right]$, then the two eigenvalues of $J_{I}$ (resp. $J_{I V}$ ) are negative. Therefore, the unique equilibrium is globally asymptotically stable.

\section{Appendix D: Dynamics in Regime II and III}

\section{1. $\quad N$ coupled nodes $-\alpha>0$}

For System (4), equilibrium points are obtained by setting $\forall i \in\{1, \cdots, N\}, \frac{d v_{i}}{d t}=0$, $\frac{d C_{K, i}}{d t}=0$ and $\frac{d C_{D, i}}{d t}=0$, that yields

$$
\frac{d v_{i}}{d t}=\frac{-1}{C_{K, i}^{*} N_{K} F_{s K} / v_{0 K, i}-C_{D, i}^{*} N_{D} F_{s D} / v_{0 D, i}-\mu} \frac{d F_{r e s, i}}{d t}=0
$$

Thus, $\frac{d F_{r e s, i}}{d t}=0$ is required. Using (B16) and (B17) that yields for $i=1$ and $i=N$, $v_{1}=v_{2}$ and $v_{N-1}=v_{N}$. When $i=\{2, \cdots, N-1\}$, using (B15) $\frac{d F_{r e s, i}}{d t}=0$ results in $v_{i}=\left(v_{i+1}+v_{i-1}\right) / 2$ (the velocity of node $i$ is equal to the mean velocity of its 2 adjacent nodes). Hence, for all $i, v_{i}^{*}=v^{*}$ where $v^{*}$ is the final filament velocity. Hence, the equilibrium values for System (4) are:

$$
\begin{array}{r}
v_{1}^{*}=v_{2}^{*}=\cdots=v_{i}^{*}=\cdots=v_{N-1}^{*}=v_{N}^{*}=v^{*} \\
\forall i, C_{K, i}^{*}=\frac{\pi_{K}}{\pi_{K}+\epsilon_{K} f_{K}\left(v^{*}\right)}=\frac{1}{1+\kappa_{K} f_{K}\left(v^{*}\right)}=C_{K}^{*}, \\
C_{D, i}^{*}=\frac{\pi_{D}}{\pi_{D}+\epsilon_{D} f_{D}\left(v^{*}\right)}=\frac{1}{1+\kappa_{D} f_{D}\left(v^{*}\right)}=C_{D}^{*}
\end{array}
$$


where $\kappa_{\bullet}=\epsilon_{\bullet} / \pi_{\bullet}$ are the desorption constants. We now characterize $v^{*}$. For all $i, \frac{d F_{r e s, i}}{d t}=0$ or $F_{\text {res }, i}=z_{i}, z_{i} \in \mathbb{R}$. From (B7) and as $\forall i, v_{i}=v^{*}$, it follows $\forall i$

$$
\begin{aligned}
F_{r e s, i}=z_{i} & =\left(1-v^{*} / v_{0 K, i}\right) C_{K, i}^{*} N_{K} F_{s K}+\left(1+v^{*} / v_{0 D, i}\right) C_{D, i}^{*} N_{D} F_{s D}+v^{*} \mu \\
& =\left(1-v^{*} / v_{0 K, i}\right) \frac{\pi_{K} N_{K} F_{s K}}{\pi_{K}+\epsilon_{K} f_{K}\left(v^{*}\right)}+\left(1+v^{*} / v_{0 D, i}\right) \frac{\pi_{D} N_{D} F_{s D}}{\pi_{D}+\epsilon_{D} f_{D}\left(v^{*}\right)}+v^{*} \mu .
\end{aligned}
$$

Furthermore, as $\forall i, v_{i}=v^{*}$ we have $v_{0 K, i}=v_{0 K}$ and $v_{0 D, i}=v_{0 D}$, it yields

$$
\sum_{i=1}^{N} F_{r e s, i}=\sum_{i=1}^{N} z_{i}=N\left[\left(1-v^{*} / v_{0 K, i}\right) \frac{\pi_{K} N_{K} F_{s K}}{\pi_{K}+\epsilon_{K} f_{K}\left(v^{*}\right)}+\left(1+v^{*} / v_{0 D, i}\right) \frac{\pi_{D} N_{D} F_{s D}}{\pi_{D}+\epsilon_{D} f_{D}\left(v^{*}\right)}+v^{*} \mu\right]
$$

where

$$
\begin{aligned}
\sum_{i=1}^{N} z_{i} & =-\alpha\left(\left(\left\|\left(x_{1}-x_{2}\right)\right\|-\ell\right) \frac{x_{1}-x_{2}}{\left\|x_{1}-x_{2}\right\|}\right) \\
& -\alpha\left(\left(\left\|\left(x_{2}-x_{1}\right)\right\|-\ell\right) \frac{x_{2}-x_{1}}{\left\|x_{2}-x_{1}\right\|}+\left(\left\|\left(x_{2}-x_{3}\right)\right\|-\ell\right) \frac{x_{2}-x_{3}}{\left\|x_{2}-x_{3}\right\|}\right) \\
& \cdots-\alpha\left(\left(\left\|\left(x_{i}-x_{i-1}\right)\right\|-\ell\right) \frac{x_{i}-x_{i-1}}{\left\|x_{i}-x_{i-1}\right\|}+\left(\left\|\left(x_{i}-x_{i+1}\right)\right\|-\ell\right) \frac{x_{i}-x_{i+1}}{\left\|x_{i}-x_{i+1}\right\|}\right) \cdots \\
& -\alpha\left(\left(\left\|\left(x_{N-1}-x_{N-2}\right)\right\|-\ell\right) \frac{x_{N-1}-x_{N-2}}{\left\|x_{N-1}-x_{N-2}\right\|}+\left(\left\|\left(x_{N-1}-x_{N}\right)\right\|-\ell\right) \frac{x_{N-1}-x_{N}}{\left\|x_{N-1}-x_{N}\right\|}\right) \\
& -\alpha\left(\left(\left\|\left(x_{N}-x_{N-1}\right)\right\|-\ell\right) \frac{x_{N}-x_{N-1}}{\left\|x_{N}-x_{N-1}\right\|}\right) \\
& =0 .
\end{aligned}
$$

Therefore, $v^{*}$ satisfies the following nonlinear equation given in $(5)$, which is

$$
0=\left(1-v^{*} / v_{0 K, i}\right) \frac{N_{K} F_{s K}}{1+\kappa_{K} f_{K}\left(v^{*}\right)}+\left(1+v^{*} / v_{0 D, i}\right) \frac{N_{D} F_{s D}}{1+\kappa_{D} f_{D}\left(v^{*}\right)}+v^{*} \mu
$$

where $\kappa_{\bullet}=\epsilon_{\bullet} / \pi_{\bullet}$. This equation is solved numerically by finding the intersections of the straight line $-v^{*} \mu$ and the curve $\left(1-v^{*} / v_{0 K, i}\right) \frac{N_{K} F_{s K}}{1+\kappa_{K} f_{K}\left(v^{*}\right)}+\left(1+v^{*} / v_{0 D, i}\right) \frac{N_{D} F_{s D}}{1+\kappa_{D} f_{D}\left(v^{*}\right)}$ in Regime II and III. Note that neither $-v_{f D}, 0$ nor $v_{f K}$, which define boundaries between regimes, satisfy (5). Hence, none of the equilibrium points are boundary equilibrium points; all are interior points.

Then, the local stability of interior equilibrium points existing in Regime II or III can be investigated by using the linearization of System (4). First of all, we note that the 
$3 N$-dimensional System (4) satisfies the following relationship: $\forall t$

$$
\begin{aligned}
\sum_{i=1}^{N}( & -\left(1-v_{i} / v_{0 K, i}\right) N_{K} F_{s K} \frac{d C_{K, i}}{d t}-\left(1+v_{i} / v_{0 D, i}\right) N_{D} F_{s D} \frac{d C_{D, i}}{d t} \\
& \left.+\left(C_{K, i} N_{K} F_{s K} / v_{0 K, i}-C_{D, i} N_{D} F_{s D} / v_{0 D, i}-\mu\right) \frac{d v_{i}}{d t}\right)=0 .
\end{aligned}
$$

Re-arranging (D2) and using $N_{\bullet} C_{\bullet}, i=n_{\bullet}, i$ yields

$$
\begin{aligned}
\sum_{i=1}^{N} \frac{d}{d t}\left[v _ { i } \left(n_{K, i} F_{s K} / v_{0 K, i}-\right.\right. & \left.\left.n_{D, i} F_{s D} / v_{0 D, i}-\mu\right)\right]-\frac{d}{d t}\left[n_{K, i} F_{s K} / v_{0 K, i}+n_{D, i} F_{s D} / v_{0 D, i}\right]=0 \\
-\frac{d}{d t}\left[\sum_{i=1}^{N}\left(1-v_{i} / v_{0 K, i}\right) n_{K, i} F_{s K}+\left(1+v_{i} / v_{0 D, i}\right) n_{D, i} F_{s D}+v_{i} \mu\right] & =0
\end{aligned}
$$

from (B7) with $a_{K, i}=a_{D, i}=1$ as in Regime II and III, (D2) can also be expressed as

$$
\frac{d}{d t}\left[\sum_{i=1}^{N} F_{r e s, i}\right]=0 .
$$

Due to this conservation law, the linearization will always have at least one zero eigenvalue.

The Jacobian matrix of System (4) in Regime II or III evaluated at the equilibrium point is a $3 N \times 3 N$ block-tridiagonal matrix

$$
J_{N}=\left[\begin{array}{ccccccc}
X & Y & 0 & \cdots & \cdots & \cdots & 0 \\
Y & X-Y & Y & \ddots & & & \vdots \\
0 & \ddots & \ddots & \ddots & \ddots & & \vdots \\
\vdots & \ddots & \ddots & \ddots & \ddots & \ddots & \vdots \\
\vdots & & \ddots & \ddots & \ddots & \ddots & 0 \\
\vdots & & & \ddots & Y & X-Y & Y \\
0 & \cdots & \cdots & \ldots & 0 & Y & X
\end{array}\right]
$$

where $X$ and $Y$ are $3 \times 3$ matrices. Due to the wild differences in magnitudes of entries, the Jacobian matrix $J_{N}$ is ill-conditioned for the numerical computations of eigenvalues. To overcome this problem, the Jacobian matrix $J_{N}$ is transformed by similarity. For any $N$, we can find a non-singular matrix $P_{N}$ such as $J_{N}$ is similar to a block-upper-triangular matrix 


\begin{tabular}{|l|l|}
\hline$N$ & $b_{i}$ in $X+b_{i} Y$ \\
\hline 2 & \pm 1 \\
3 & $1,0,-2$ \\
4 & $\pm 1,-(1 \pm \sqrt{2})$ \\
5 & $1,-\left(\frac{3 \pm \sqrt{5}}{2}\right),-\left(\frac{1 \pm \sqrt{5}}{2}\right)$ \\
6 & $\pm 1,0,-2,-(1 \pm \sqrt{3})$ \\
\hline
\end{tabular}

TABLE IV. Coefficients $b_{i}$ with $i \in\{1,2, \cdots, N\}$ for $N=2,3,4,5,6$. Recall that $b_{1}=1$.

$T_{N}$

$$
P_{N}^{-1} J_{N} P_{N}=T_{N}=\left[\begin{array}{ccccc}
X+b_{1} Y & Y & 0 & \cdots & 0 \\
0 & X+b_{2} Y & Y & \ddots & \vdots \\
\vdots & \ddots & \ddots & \ddots & 0 \\
\vdots & & \ddots & \ddots & Y \\
0 & \cdots & \cdots & 0 & X+b_{N} Y
\end{array}\right]
$$

where $b_{i}$ are real scalar. The algorithm to compute $T_{N}$ is given in Appendix E. Values of coefficients $b_{i}$ are given for some $N$ in Table IV.

Hence, as $J_{N}$ and $T_{N}$ have the same eigenvalues, the stability of equilibria can be discussed in terms of signs of the real parts of eigenvalues of $N 3 \times 3$ matrices having the form $X+b_{i} Y$ with $b_{i} \in \mathbb{R}$ instead of the signs of the real parts of eigenvalues of a $3 N \times 3 N$ matrix.

The $N$ matrices $X+b_{i} Y$ with $b_{i} \in \mathbb{R}$ take the form

$$
X+b_{i} Y=\left[\begin{array}{ccc}
M_{1} & 0 & M_{2} \\
0 & M_{3} & M_{4} \\
M_{5} & M_{6} & M_{7}+b_{i} M_{8}
\end{array}\right] \text { where } X=\left[\begin{array}{ccc}
M_{1} & 0 & M_{2} \\
0 & M_{3} & M_{4} \\
M_{5} & M_{6} & M_{7}
\end{array}\right] \text { and } Y=\left[\begin{array}{ccc}
0 & 0 & 0 \\
0 & 0 & 0 \\
0 & 0 & M_{8}
\end{array}\right]
$$

and their characteristic polynomial is

$$
\begin{aligned}
P_{X+b_{i} Y}(\lambda) & =\lambda^{3}-\left[M_{1}+M_{3}+M_{7}+b_{i} M_{8}\right] \lambda^{2} \\
& +\left[M_{1}\left(M_{3}+M_{7}+b_{i} M_{8}\right)+M_{3}\left(M_{7}+b_{i} M_{8}\right)-M_{4} M_{6}-M_{2} M_{5}\right] \lambda \\
& -M_{1} M_{3}\left(M_{7}+b_{i} M_{8}\right)+M_{1} M_{4} M_{6}+M_{2} M_{3} M_{5}
\end{aligned}
$$


with

$$
\begin{array}{rr}
M_{1}=-\pi_{K}-\epsilon_{K} f_{K}\left(v^{*}\right)<0, & M_{2}=-\epsilon_{K} \frac{d f_{K}}{d v}\left(v^{*}\right) C_{K}^{*} \\
M_{3}=-\pi_{D}-\epsilon_{D} f_{D}\left(v^{*}\right)<0, & M_{4}=-\epsilon_{D} \frac{d f_{D}}{d v}\left(v^{*}\right) C_{D}^{*} \\
M_{5}=N_{K} F_{s K}\left(1-v^{*} / v_{0 K, i}\right) \frac{M_{1}}{M}, & M_{6}=N_{D} F_{s D}\left(1+v^{*} / v_{0 D, i}\right) \frac{M_{3}}{M} \\
M_{7}=\left(\frac{\alpha_{1}}{N}+N_{K} F_{s K}\left(1-v^{*} / v_{0 K, i}\right) M_{2}+N_{D} F_{s D}\left(1+v^{*} / v_{0 D, i}\right) M_{4}\right) \frac{1}{M}, & M_{8}=-\frac{\alpha_{1}}{N} \frac{1}{M}>0 \\
M=N_{K} F_{s K} / v_{0 K, i} C_{K}^{*}-N_{D} F_{s D} / v_{0 D, i} C_{D}^{*}-\mu<0 &
\end{array}
$$

where $\alpha=\frac{\alpha_{1}}{N}$ and

$$
\frac{d f_{K}}{d v}\left(v^{*}\right)=\frac{\partial f_{K}}{\partial v_{1}}\left(v^{*}\right)=\cdots=\frac{\partial f_{K}}{\partial v_{N}}\left(v^{*}\right), \quad \frac{d f_{D}}{d v}\left(v^{*}\right)=\frac{\partial f_{D}}{\partial v_{1}}\left(v^{*}\right)=\cdots=\frac{\partial f_{D}}{\partial v_{N}}\left(v^{*}\right)
$$

Using Routh-Hurwitz criterion on the $N$ characteristic polynomials (D3) yields the $3 N$ eigenvalues of $J_{N}$ have negative real part if and only if the 3 following conditions are satisfied for all $b_{i}$ with $i \in\{1,2, \cdots, N\}$

$$
\begin{aligned}
& M_{1}+M_{3}+M_{7}+b_{i} M_{8}<0, \\
&-M_{1} M_{3}\left(M_{7}+b_{i} M_{8}\right)+M_{1} M_{4} M_{6}+M_{2} M_{3} M_{5}>0, \\
&-\left[M_{1}+M_{3}+M_{7}+b_{i} M_{8}\right] \\
& \times\left[M_{1}\left(M_{3}+M_{7}+b_{i} M_{8}\right)+M_{3}\left(M_{7}+b_{i} M_{8}\right)-M_{4} M_{6}-M_{2} M_{5}\right]>-M_{1} M_{3}\left(M_{7}+b_{i} M_{8}\right) \\
&+M_{1} M_{4} M_{6}+M_{2} M_{3} M_{5} .
\end{aligned}
$$

Note that the second and third condition can be simplified as follows:

$$
\begin{gathered}
\left(1-b_{i}\right) M_{1} M_{3} M_{8}>0 \\
-\left[M_{1}+M_{3}+M_{7}+b_{i} M_{8}\right] \\
\times\left[M_{1}\left(M_{3}+M_{7}+b_{i} M_{8}\right)+M_{3}\left(M_{7}+b_{i} M_{8}\right)-M_{4} M_{6}-M_{2} M_{5}\right]>\left(1-b_{i}\right) M_{1} M_{3} M_{8} .
\end{gathered}
$$

For any number of node $N, b_{1}=1$ (see Appendix E); then, the second condition is never satisfied for the first $3 \times 3$ matrix, $X+b_{1} Y$. Recall that due to the conservation law, there is one zero eigenvalue. Hence, an equilibrium point $\left(C_{K}^{*}, C_{D}^{*}, v^{*}\right)$ is locally asymptotically 
stable when the following conditions are satisfied

for $X+b_{1} Y=X+Y$,

$$
\begin{array}{r}
M_{1}+M_{3}+M_{7}+M_{8}<0, \\
-\left[M_{1}+M_{3}+M_{7}+M_{8}\right] \\
\times\left[M_{1}\left(M_{3}+M_{7}+M_{8}\right)+M_{3}\left(M_{7}+M_{8}\right)-M_{4} M_{6}-M_{2} M_{5}\right]>0,
\end{array}
$$

for all $X+b_{i} Y$ with $i \in\{2, \ldots, N\}$,

$$
\begin{gathered}
M_{1}+M_{3}+M_{7}+b_{i} M_{8}<0, \\
\left(1-b_{i}\right) M_{1} M_{3} M_{8}>0, \\
-\left[M_{1}+M_{3}+M_{7}+b_{i} M_{8}\right] \\
\times\left[M_{1}\left(M_{3}+M_{7}+b_{i} M_{8}\right)+M_{3}\left(M_{7}+b_{i} M_{8}\right)-M_{4} M_{6}-M_{2} M_{5}\right]>\left(1-b_{i}\right) M_{1} M_{3} M_{8} .
\end{gathered}
$$

Remark that, as $M_{1} M_{3} M_{8}>0$, if any value $b_{i}$ does not satisfy $1 \geq b_{i}$, all the equilibria are unstable. Note that from Table IV this condition is verified for all $b_{i}$ computed for $N=2,3,4,5,6$. The length and elastic properties of filaments are involved in the stability conditions through $M_{7}+b_{i} M_{8}$.

\section{2. $\quad N$ uncoupled nodes $-\alpha=0$}

The equilibrium points are the same as for $\alpha>0$ (coupled nodes). The Jacobian matrix $J_{N}$ is a block diagonal

$$
J_{N}=\operatorname{diag}(\tilde{X})
$$

where

$$
\tilde{X}=\left[\begin{array}{ccc}
M_{1} & 0 & M_{2} \\
0 & M_{3} & M_{4} \\
M_{5} & M_{6} & \tilde{M}_{7}
\end{array}\right]
$$

with

$$
\tilde{M}_{7}=\left(N_{K} F_{s K}\left(1-v^{*} / v_{0 K, i}\right) M_{2}+N_{D} F_{s D}\left(1+v^{*} / v_{0 D, i}\right) M_{4}\right) \frac{1}{M} .
$$

In other words, $\tilde{X}=X+Y=X+b_{1} Y$. Thus, an equilibrium point $\left(C_{K}^{*}, C_{D}^{*}, v^{*}\right)$ is locally asymptotically stable if and only if the conditions given in (D4) are satisfied for $b_{1}=1$.

Hence, an equilibrium point that is unstable in the uncoupled case will be unstable in the coupled case. For an equilibrium point that is locally asymptotically stable in the uncoupled 
case, $3(N-1)$ extra conditions ((D4) for $b_{i}$ with $\left.i \in\{2, \cdots, N\}\right)$ will have to be satisfied to maintain the local asymptotic stability in the coupled case. These conditions depend on the length and elastic properties of filaments.

\section{Appendix E: Similar matrices}

Algorithm to find a block-upper-triangular matrix $T_{N}$ similar to the $3 N \times 3 N$ Jacobian matrix $J_{N}$ : find $P_{N}$ such that $T_{N}=P_{N}^{-1} J_{N} P_{N}$. Example given for $N=6$.

1. Initialization: Start with a $3 N \times 3 N$ matrix $P_{N}$ with the following form

$$
P_{N}=\left[\begin{array}{cccccc}
I & 0 & 0 & 0 & 0 & 0 \\
I & I & 0 & 0 & 0 & 0 \\
I & p_{2} I & I & 0 & 0 & 0 \\
I & p_{4} I & p_{5} I & I & 0 & 0 \\
I & p_{7} I & p_{8} I & p_{9} I & I & 0 \\
I & p_{11} I & p_{12} I & p_{13} I & p_{14} I & I
\end{array}\right]
$$

where $I$ is the $3 \times 3$ identity matrix.

Compute $P_{N}^{-1}$ and $P_{N}^{-1} J_{N} P_{N}=T_{N}$

$$
T_{N}=\left[\begin{array}{cccccc}
X+Y & Y & 0 & 0 & 0 & 0 \\
0 & X+\left(p_{2}-1-1\right) Y & Y & 0 & 0 & 0 \\
0 & f_{2,1}\left(p_{i}\right) Y & X+\left(p_{5}-p_{2}-1\right) Y & Y & 0 & 0 \\
0 & f_{2,2}\left(p_{i}\right) Y & f_{5,1}\left(p_{i}\right) Y & X+\left(p_{9}-p_{5}-1\right) Y & Y & 0 \\
0 & f_{2,3}\left(p_{i}\right) Y & f_{5,2}\left(p_{i}\right) Y & f_{9,1}\left(p_{i}\right) Y & X+\left(p_{14}-p_{9}-1\right) Y & Y \\
0 & f_{2,4}\left(p_{i}\right) Y & f_{5,3}\left(p_{i}\right) Y & f_{9,2}\left(p_{i}\right) Y & f_{14,1}\left(p_{i}\right) Y & X-p_{14} Y
\end{array}\right]
$$

2. Work on Column 2 to characterize $p_{2} \in \mathbb{R}$

(a) $f_{2,1}\left(p_{i}\right)=\left(p_{4}-p_{2}\left(p_{2}-1\right)\right)$. Set $p_{4}=p_{2}\left(p_{2}-1\right)$ to get $f_{2,1}\left(p_{i}\right)=0$; now $f_{2,2}\left(p_{i}\right)=$ $p_{7}-\left(p_{2}^{2}\left(p_{2}-2\right)+1\right)$

$$
T_{N}=\left[\begin{array}{cccccc}
X+Y & Y & 0 & 0 & 0 & 0 \\
0 & X+\left(p_{2}-1-1\right) Y & Y & 0 & 0 & 0 \\
0 & 0 & X+\left(p_{5}-p_{2}-1\right) Y & Y & 0 & 0 \\
0 & \left(p_{7}-\left(p_{2}^{2}\left(p_{2}-2\right)+1\right)\right) Y & f_{5,1}\left(p_{i}\right) Y & X+\left(p_{9}-p_{5}-1\right) Y & Y & 0 \\
0 & f_{2,3}\left(p_{i}\right) Y & f_{5,2}\left(p_{i}\right) Y & f_{9,1}\left(p_{i}\right) Y & X+\left(p_{14}-p_{9}-1\right) Y & Y \\
0 & f_{2,4}\left(p_{i}\right) Y & f_{5,3}\left(p_{i}\right) Y & f_{9,2}\left(p_{i}\right) Y & f_{14,1}\left(p_{i}\right) Y & X-p_{14} Y
\end{array}\right]
$$

(b) Set $p_{7}=p_{2}^{2}\left(p_{2}-2\right)+1$ to make $f_{2,2}\left(p_{i}\right)=0$ and obtain $f_{2,3}\left(p_{i}\right)=p_{11}-\left(p_{2}^{4}-\right.$ $\left.3 b_{2}^{3}+b_{2}^{2}+2 b_{2}\right)$

(c) Set $p_{11}=p_{2}^{4}-3 b_{2}^{3}+b_{2}^{2}+2 b_{2}$ to make $f_{2,3}\left(p_{i}\right)=0$ and $f_{2,4}\left(p_{2}\right)=-p_{2}^{5}+5 p_{2}^{4}-$ $6 p_{2}^{3}-2 p_{2}^{2}+4 p_{2}$ a polynomial in $p_{2}$. Find roots of $f_{2,4}\left(p_{2}\right)=0$. Pick a real root of $f_{2,4}\left(p_{2}\right)=0$ for $p_{2}$. All roots might not be suitable, as they might make the next polynomial irreducible.

$$
T_{N}=\left[\begin{array}{cccccc}
X+Y & Y & 0 & 0 & 0 & 0 \\
0 & X+\left(p_{2}-1-1\right) Y & Y & 0 & 0 & 0 \\
0 & 0 & X+\left(p_{5}-p_{2}-1\right) Y & Y & 0 & 0 \\
0 & 0 & f_{5,1}\left(p_{i}\right) Y & X+\left(p_{9}-p_{5}-1\right) Y & Y & 0 \\
0 & 0 & f_{5,2}\left(p_{i}\right) Y & f_{9,1}\left(p_{i}\right) Y & X+\left(p_{14}-p_{9}-1\right) Y & Y \\
0 & 0 & f_{5,3}\left(p_{i}\right) Y & f_{9,2}\left(p_{i}\right) Y & f_{14,1}\left(p_{i}\right) Y & X-p_{14} Y
\end{array}\right]
$$


3. Work on Column 3 to characterize $p_{5}$

(a) To make $f_{5,1}\left(p_{i}\right)=0$, replace $p_{8}$ as a function of $p_{5}$.

(b) To make $f_{5,2}\left(p_{i}\right)=0$, replace $p_{12}$ as a function of $p_{5}$.

(c) Now $f_{5,3}\left(p_{5}\right)$ is a polynomial of $p_{5}$; solve $f_{5,3}\left(p_{5}\right)=0$ for $p_{5}$. If $f_{5,3}\left(p_{5}\right)$ is composed of irreducible $2^{\text {nd }}$-order polynomial, go back to $2 \mathrm{c}$ ) and try with another real root of $f_{2,4}\left(p_{2}\right)=0$ for $p_{2}$. Pick a real root of $f_{5,3}\left(p_{5}\right)=0$ for $p_{5}$. All roots might not be suitable, as they might make the next polynomial irreducible.

$$
T_{N}=\left[\begin{array}{cccccc}
X+Y & Y & 0 & 0 & 0 & 0 \\
0 & X+\left(p_{2}-1-1\right) Y & Y & 0 & 0 & 0 \\
0 & 0 & X+\left(p_{5}-p_{2}-1\right) Y & Y & 0 & 0 \\
0 & 0 & 0 & X+\left(p_{9}-p_{5}-1\right) Y & Y & 0 \\
0 & 0 & 0 & f_{9,1}\left(p_{i}\right) Y & X+\left(p_{14}-p_{9}-1\right) Y & Y \\
0 & 0 & 0 & f_{9,2}\left(p_{i}\right) Y & f_{14,1}\left(p_{i}\right) Y & X-p_{14} Y
\end{array}\right]
$$

4. Work on Column 4 to characterize $p_{9}$

(a) To make $f_{9,1}\left(p_{i}\right)=0$, replace $p_{13}$ as a function of $p_{9}$. Obtain $f_{9,2}\left(p_{9}\right)$ as a polynomial in $p_{9}$.

(b) Solve $f_{9,2}\left(p_{9}\right)=0$ for $p_{9}$. If $f_{9,2}\left(p_{9}\right)$ is composed of irreducible $2^{\text {nd }}$-order polynomial, go back to $3 \mathrm{c}$ ) and try with another real root of $f_{5,3}\left(p_{5}\right)=0$ for $p_{5}$.

(c) Pick a real root of $f_{9,2}\left(p_{9}\right)=0$ for $p_{9}$. All roots might not be suitable, as they might make the next polynomial irreducible.

$$
T_{N}=\left[\begin{array}{cccccc}
X+Y & Y & 0 & 0 & 0 & 0 \\
0 & X+\left(p_{2}-1-1\right) Y & Y & 0 & 0 & 0 \\
0 & 0 & X+\left(p_{5}-p_{2}-1\right) Y & Y & 0 & 0 \\
0 & 0 & 0 & X+\left(p_{9}-p_{5}-1\right) Y & Y & 0 \\
0 & 0 & 0 & 0 & X+\left(p_{14}-p_{9}-1\right) Y & Y \\
0 & 0 & 0 & 0 & f_{14,1}\left(p_{i}\right) Y & X-p_{14} Y
\end{array}\right]
$$

5. Work of Column 5 to characterize $p_{14}$

(a) Solve $f_{14,1}\left(p_{14}\right)=0$ which is a polynomial equation in $p_{14}$. If $f_{14,1}\left(p_{14}\right)$ is composed of irreducible $2^{\text {nd }}$-order polynomial, go back to $4 \mathrm{c}$ ) and try with another real root of $f_{9,2}\left(p_{9}\right)=0$ for $p_{9}$.

(b) Pick a real root of $f_{14,1}\left(p_{14}\right)=0$ for $p_{14}$.

$$
T_{N}=\left[\begin{array}{cccccc}
X+Y & Y & 0 & 0 & 0 & 0 \\
0 & X+\left(p_{2}-1-1\right) Y & Y & 0 & 0 & 0 \\
0 & 0 & X+\left(p_{5}-p_{2}-1\right) Y & Y & 0 & 0 \\
0 & 0 & 0 & X+\left(p_{9}-p_{5}-1\right) Y & Y & 0 \\
0 & 0 & 0 & 0 & X+\left(p_{14}-p_{9}-1\right) Y & Y \\
0 & 0 & 0 & 0 & 0 & X-p_{14} Y
\end{array}\right]
$$

To summarize, to characterize $P_{N}$ and compute $T_{N}$ only $p_{2}, p_{5}, p_{9}, p_{14}, p_{20}, \cdots, p_{\sum_{i=3}^{N} i-1}$ have to be found as real roots of some polynomials. The coefficients $b_{i}$ in $T_{N}$ are: $b_{1}=1$, 
$b_{2}=p_{2}-1-1, b_{3}=p_{5}-p_{2}-1, b_{4}=p_{9}-p_{5}-1, b_{i}=p_{\sum_{j=3}^{i+1} j-1}-p_{\sum_{j=3}^{i} j-1}-1$ and $b_{N}=-p_{\sum_{i=3}^{N} i-1}$. Values for $b_{i}$ obtained with the algorithm are provided in Table IV for $N=2,3,4,5,6$.

For $N=2$, the Jacobian matrix $J_{2}$ takes the form of the block matrix

$$
J_{2}=\left[\begin{array}{ll}
X & Y \\
Y & X
\end{array}\right]
$$

and $J_{2}$ is similar to the matrix

$$
T_{2}=\left[\begin{array}{cc}
X+Y & Y \\
0 & X-Y
\end{array}\right]=P_{2}^{-1} J_{2} P_{2}=\left[\begin{array}{cc}
I & 0 \\
-I & I
\end{array}\right]\left[\begin{array}{cc}
X & Y \\
Y & X
\end{array}\right]\left[\begin{array}{cc}
I & 0 \\
I & I
\end{array}\right] .
$$

By similarity, the $6 \times 6$ matrix $J_{2}$ has the same eigenvalues of the two $3 \times 3$ matrices, $X+Y$ and $X-Y$.

For $N=3$, the Jacobian matrix is

$$
J_{3}=\left[\begin{array}{ccc}
X & Y & 0 \\
Y & X-Y & Y \\
0 & Y & X
\end{array}\right]
$$

which is similar to

$$
T_{3}=\left[\begin{array}{ccc}
X+Y & Y & 0 \\
0 & X & Y \\
0 & 0 & X-2 Y
\end{array}\right]=\left[\begin{array}{ccc}
I & 0 & 0 \\
-I & I & 0 \\
I & -2 I & I
\end{array}\right]\left[\begin{array}{ccc}
X & Y & 0 \\
Y & X-Y & Y \\
0 & Y & X
\end{array}\right]\left[\begin{array}{ccc}
I & 0 & 0 \\
I & I & 0 \\
I & 2 I & I
\end{array}\right] .
$$

By similarity, the $9 \times 9$ matrix $J_{3}$ has the same eigenvalues of the three following $3 \times 3$ matrices $X, X+Y$ and $X-2 Y$.

For $N=4$, the Jacobian matrix is

$$
J_{4}=\left[\begin{array}{cccc}
X & Y & 0 & 0 \\
Y & X-Y & Y & 0 \\
0 & Y & X-Y & Y \\
0 & 0 & Y & X
\end{array}\right]
$$

which is similar to

$$
T_{4}=\left[\begin{array}{cccc}
X+Y & Y & 0 & 0 \\
0 & X-Y & Y & 0 \\
0 & 0 & X-(1-\sqrt{2}) Y & Y \\
0 & 0 & 0 & X-(1+\sqrt{2}) Y
\end{array}\right]=P_{4}^{-1} J_{4} P_{4}
$$


with

$$
P_{4}=\left[\begin{array}{cccr}
I & 0 & 0 & 0 \\
I & I & 0 & 0 \\
I & I & I & 0 \\
I & 0 & (1+\sqrt{2}) I & I
\end{array}\right] .
$$

By similarity, the $12 \times 12$ matrix $J_{4}$ has the same eigenvalues of the four following $3 \times 3$ matrices $X+Y, X-Y$, and $X-(1 \pm \sqrt{2}) Y$.

For $N=5$ the Jacobian matrix is

$$
J_{5}=\left[\begin{array}{ccccc}
X & Y & 0 & 0 & 0 \\
Y & X-Y & Y & 0 & 0 \\
0 & Y & X-Y & Y & 0 \\
0 & 0 & Y & X-Y & Y \\
0 & 0 & 0 & Y & X
\end{array}\right]
$$

which is similar to

$$
T_{5}=\left[\begin{array}{ccccc}
X+Y & Y & 0 & 0 & 0 \\
0 & X-\frac{3-\sqrt{5}}{2} Y & Y & 0 & 0 \\
0 & 0 & X-\frac{1-\sqrt{5}}{2} Y & Y & 0 \\
0 & 0 & 0 & X-\frac{3+\sqrt{5}}{2} Y & Y \\
0 & 0 & 0 & 0 & X-\frac{1+\sqrt{5}}{2} Y
\end{array}\right]=P_{5}^{-1} J_{5} P_{5}
$$

with

$$
P_{5}=\left[\begin{array}{ccccc}
I & 0 & 0 & 0 & 0 \\
I & I & 0 & 0 & 0 \\
I & \frac{1+\sqrt{5}}{2} I & I & 0 & 0 \\
I & I & (1+\sqrt{5}) I & I & 0 \\
I & -I & (3+\sqrt{5}) I & \frac{1+\sqrt{5}}{2} I & I
\end{array}\right]
$$

For $N=6$ the Jacobian matrix is

$$
J_{6}=\left[\begin{array}{cccccc}
X & Y & 0 & 0 & 0 & 0 \\
Y & X-Y & Y & 0 & 0 & 0 \\
0 & Y & X-Y & Y & 0 & 0 \\
0 & 0 & Y & X-Y & Y & 0 \\
0 & 0 & 0 & Y & X-Y & Y \\
0 & 0 & 0 & 0 & Y & X
\end{array}\right]
$$


which is similar to

$$
T_{6}=\left[\begin{array}{cccccc}
X+Y & Y & 0 & 0 & 0 & 0 \\
0 & X-Y & Y & 0 & 0 & 0 \\
0 & 0 & X & Y & 0 & 0 \\
0 & 0 & 0 & X-2 Y & Y & 0 \\
0 & 0 & 0 & 0 & X-(1-\sqrt{3}) Y & Y \\
0 & 0 & 0 & 0 & 0 & X-(1+\sqrt{3}) Y
\end{array}\right]=P_{6}^{-1} J_{6} P_{6}
$$

with

$$
P_{6}=\left[\begin{array}{cccccc}
I & 0 & 0 & 0 & 0 & 0 \\
I & I & 0 & 0 & 0 & 0 \\
I & I & I & 0 & 0 & 0 \\
I & 0 & 2 I & I & 0 & 0 \\
I & 0 & I & I & I & 0 \\
I & I & -I & -I(\sqrt{3}+1) I & I
\end{array}\right]
$$

\section{Appendix F: Numerical bifurcation analysis in Regime II and III}

Here, System (4) in Regime II and III is studied in isolation (without taking into account the consequences of trapping in Regime I or IV). Using the local stability analysis described in Appendix C, a numerical bifurcation analysis is carried out to characterize the effects of changes in the motor strength $\left|F_{s \bullet}\right| / F_{d \bullet}$ (Table V). In first column, it is assumed that both types of motors have exponential off rates; in the second column, dyneins have catch-bond and kinesins have exponential off rates. In the first row, the case of $N=5$ uncoupled nodes is presented; in the second row, there are filaments composed of 5 coupled nodes. Note that results are the same for $N$ uncoupled nodes and $N$ coupled nodes.

In Table $\mathrm{V}$, nine figures are presented (See online version for colour):

- in the first row, there are three $3 D$ diagrams of bifurcation: the equilibrium values $C_{K}^{*}-C_{D}^{*}, v^{*}$ and a zoom in of $v^{*}$ around zero as functions of motor strengths;

- in the second row, 2D-projections of $3 D$ diagrams to the kinesin parameter plane: the first 2D-projection shows $C_{K}^{*}-C_{D}^{*}$ and the second is a zoom in around zero of $v^{*}$. In the last column, histogram of stable equilibrium velocities $v^{*}$ obtained as varying motor strengths; 
- in the third row, 2D-projections of $3 D$ diagrams to the dynein parameter plane: the first 2D-projection shows $C_{K}^{*}-C_{D}^{*}$ and the second is about $v^{*}$. In the last row, the phase diagram is a 2D-projection on the parameter space of the number of locally asymptotically stable equilibrium points depending on parameter values. These figures are the same as Figure 3.

Unstable equilibrium points are represented in gray, locally asymptotically stable equilibrium points are represented in magenta (resp. cyan) when the final velocity is positive (resp. negative). The black line represents the zero velocity (the switching hyperplane, $v_{i}=0$ ).

\section{Appendix G: Numerical simulations of the complete model}

Figure 15 shows results similar to Figure 8 run with a longer final time $t_{\text {final }}=180 \mathrm{~s}$.

\section{ACKNOWLEDGMENTS}

The authors would like to thank the Isaac Newton Institute for Mathematical Sciences for support and hospitality during the programme "Coupling Geometric PDE's with Physics for Cell Morphology, Motility and Pattern Formation" when the work on this paper was undertaken. SP would like to thank Institut Pasteur for support of her stay in Fall 2016. SP would like to thank Pauline van den Driessche for discussions on similar matrices. SP is

partly supported by an NSERC Discovery Grant. SE-M and CL were supported in part by EPSRC grant numbers EP/K032208/1 and EP/R014604/1, La Ligue contre le cancer and the Institut Pasteur.

[1] S. Portet, N. Mücke, R. Kirmse, J. Langowski, M. Beil, and H. Herrmann, Langmuir 25, 8817 (2009).

[2] S. Portet, Journal of Theoretical Biology 332, 20 (2013).

[3] S. Köster, D. A. Weitz, R. D. Goldman, U. Aebi, and H. Herrmann, Current Opinion in Cell Biology 32, 82 (2015). 


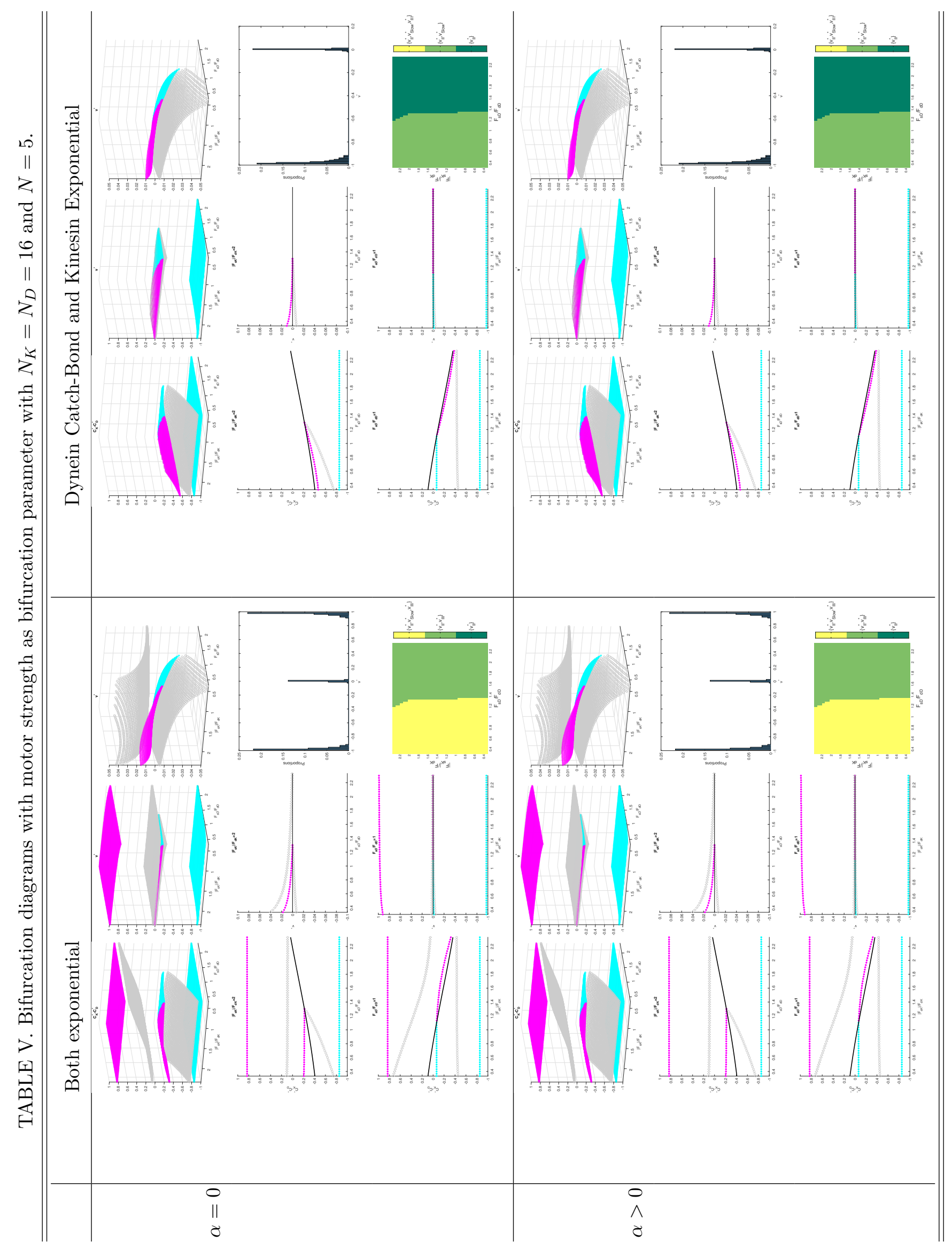




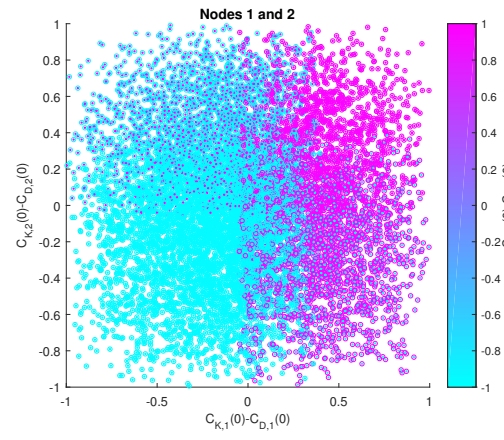

(a) $\alpha=0$

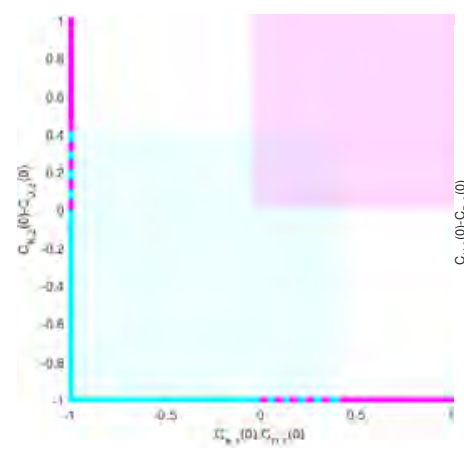

(c) Implicit coordination

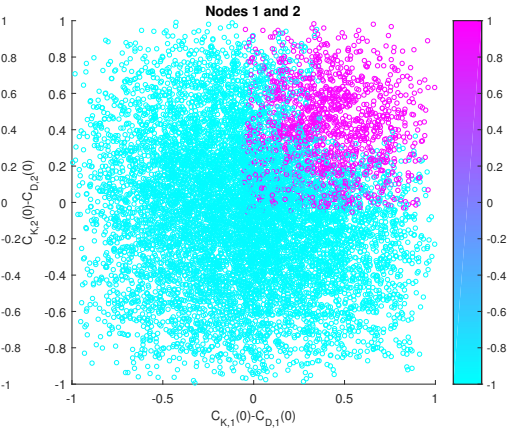

(b) $10^{-3} \alpha$

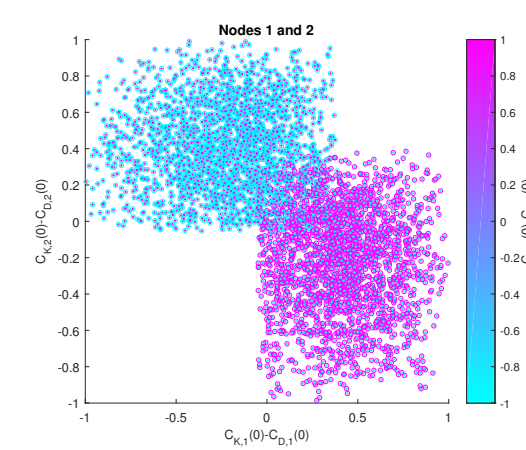

(e) $\alpha=0$

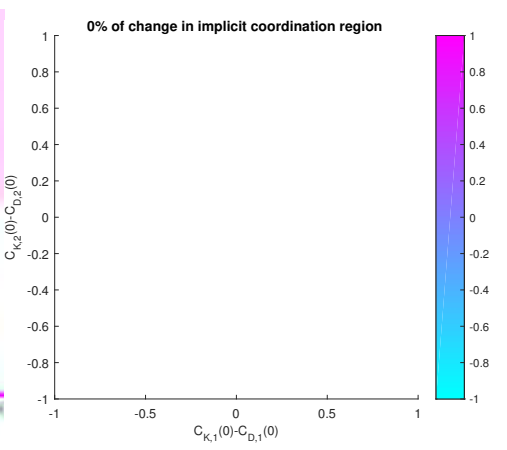

(d) $10^{-3} \alpha$

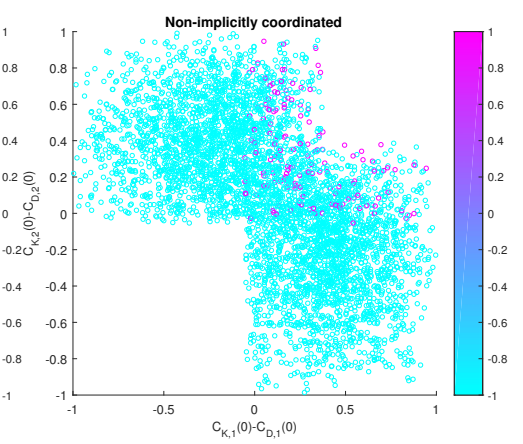

(f) $10^{-3} \alpha$

FIG. 15. Same conditions (filament of length $N=2$ with $N_{K}=N_{D}=4$ in Case 1) as in Figure 8 run for longer final time $t_{\text {final }}=180 \mathrm{~s}$. (15a-15b) 10,000 simulations with initial conditions $C_{K_{i}}(0)$ and $C_{D_{i}}(0)$ uniformly distributed on $[0,1]$ are performed; for $10^{-3} \alpha$, only simulations terminating with both nodes at the same final velocity are considered $\left(\left|v_{1}\left(t_{\text {final }}\right)-v_{2}\left(t_{\text {final }}\right)\right|<10^{-3}\right)$. (15a and 15e) For uncoupled nodes, outer (resp. inner) circle represents node 1 (resp. node 2). (15d) No changes in regions of implicit coordination resulting from elastic coupling is observed. (15e-15f) Dynamics in non-implicitly coordinated regions. See online version for colour. 
[4] J. Block, H. Witt, A. Candelli, E. J. Peterman, G. J. Wuite, A. Janshoff, and S. Köster, Physical Review Letters 118, 048101 (2017).

[5] E. Charrier and P. Janmey, Methods in Enzymology 568, 35 (2017).

[6] N. Mücke, L. Kreplak, R. Kirmse, T. Wedig, H. Herrmann, U. Aebi, and J. Langowski, Journal of Molecular Biology 335, 1241 (2004).

[7] L. Kreplak and D. Fudge, BioEssays 29, 26 (2007).

[8] J.-F. Nolting, W. Möbius, and S. Köster, Biophysical Journal 107, 2693 (2014).

[9] S. Etienne-Manneville, Annual Review in Cell and Developmental Biology 34 (2018).

[10] A. Robert, C. Hookway, and V. I. Gelfand, BioEssays 38, 232 (2016).

[11] B. T. Helfand, L. Chang, and R. D. Goldman, Journal of Cell Science 117, 133 (2004).

[12] C. Hookway, L. Ding, M. W. Davidson, J. Z. Rappoport, G. Danuser, and V. I. Gelfand, Molecular Biology of the Cell 26, 1675 (2015).

[13] N. T. Snider and M. B. Omary, Nature Reviews Molecular Cell Biology 15, 163 (2014).

[14] C. Leduc and S. Etienne-Manneville, Journal of Cell Biology 216, 1689 (2017).

[15] R. E. Leube, M. Moch, and R. Windoffer, Cold Spring Harbor Perspectives in Biology 9, a021980 (2017).

[16] J. D. Fenn, C. M. Johnson, J. Peng, P. Jung, and A. Brown, Cytoskeleton 75, 22 (2018).

[17] A. Robert, P. Tian, S. A. Adam, M. Kittisopikul, K. Jaqaman, R. D. Goldman, and V. I. Gelfand, FASEB Journal 33, 00 (2019).

[18] M. J. Müller, S. Klumpp, and R. Lipowsky, Proceedings of the National Academy of Sciences 105, 4609 (2008).

[19] M. J. Müller, S. Klumpp, and R. Lipowsky, Biophysical Journal 98, 2610 (2010).

[20] W. O. Hancock, Nature Reviews Molecular Cell Biology 15, 615 (2014).

[21] S. Klein, C. Appert-Rolland, and L. Santen, Europhysics Letters 111, 68005 (2015).

[22] J. Dallon, C. Leduc, S. Etienne-Manneville, and S. Portet, Journal of Theoretical Biology 464, 132 (2019).

[23] N. Carter and R. Cross, Nature 435, 308 (2005).

[24] A. Kunwar and A. Mogilner, Physical Biology 7, 16012 (2010).

[25] J. G. De La Torre and C. Lopez, Biopolymers 23, 611 (1984).

[26] A. Kunwar, S. K. Tripathy, J. Xu, M. K. Mattson, P. Anand, R. Sigua, M. Vershinin, R. J. McKenney, C. Y. Clare, A. Mogilner, et al., Proceedings of the National Academy of Sciences 
108, 18960 (2011).

[27] A. Takshak and A. Kunwar, Protein Science 25, 1075 (2016).

[28] C. Leidel, R. A. Longoria, F. M. Gutierrez, and G. T. Shubeita, Biophysical Journal 103, $492(2012)$.

[29] A. K. Rai, A. Rai, A. J. Ramaiya, R. Jha, and R. Mallik, Cell 152, 172 (2013).

[30] M. P. Nicholas, F. Berger, L. Rao, S. Brenner, C. Cho, and A. Gennerich, Proceedings of the National Academy of Sciences 112, 6371 (2015).

[31] S. Klumpp and R. Lipowsky, Proceedings of the National Academy of Sciences 102, 17284 (2005).

[32] Z. Qin, C.-C. Chou, L. Kreplak, and M. Buehler, in Advances in Cell Mechanics, edited by S. Li and B. Sun (Springer, 2011) Chap. 4, pp. 139-140.

[33] C. Guzman, S. Jeney, L. Kreplak, S. Kasas, A. Kulik, U. Aebi, and L. Forro, Journal of Molecular Biology 360, 623 (2006). 